\title{
An animated picture says at least a thousand words: Selecting Gif-based Replies in Multimodal Dialog
}

\author{
Xingyao Wang \\ University of Michigan \\ xingyaow@umich.edu
}

\author{
David Jurgens \\ University of Michigan \\ jurgens@umich.edu
}

\begin{abstract}
Online conversations include more than just text. Increasingly, image-based responses such as memes and animated gifs serve as culturally recognized and often humorous responses in conversation. However, while NLP has broadened to multimodal models, conversational dialog systems have largely focused only on generating text replies. Here, we introduce a new dataset of $1.56 \mathrm{M}$ text-gif conversation turns and introduce a new multimodal conversational model PEPE THE KING PRAWN for selecting gif-based replies. We demonstrate that our model produces relevant and high-quality gif responses and, in a large randomized control trial of multiple models replying to real users, we show that our model replies with gifs that are significantly better received by the community.
\end{abstract}

\section{Introduction}

Conversations are central to many online social platforms. While most conversations are textbased, computer mediated dialog also affords alternative forms of communication, such as emoji or stickers like bitmoji, that allow users to express themselves (Tang and Hew, 2019; Konrad et al., 2020). Increasingly, these visual forms of communication have become common in social media (Bourlai and Herring, 2014; Highfield and Leaver, 2016), with a notable use of the reaction gif (Bakhshi et al., 2016; Miltner and Highfield, 2017). These gifs are short video sequences that depict a particular scene and sometimes contain text that acts as a meta-commentary (Eppink, 2014). As a result, conversations become multimodal where individuals reply to one another using combinations of text and gifs (Figure 1). While conversational AI systems have been developed in a purely textbased setting, such systems do not capture the full multimodal behavior seen online. Here, we study multimodal conversation by introducing new dialog models for selecting gif replies in conversation.
PizzaMagic: Ahhhhh!!! The EMNLP deadline is in 24 hours!!

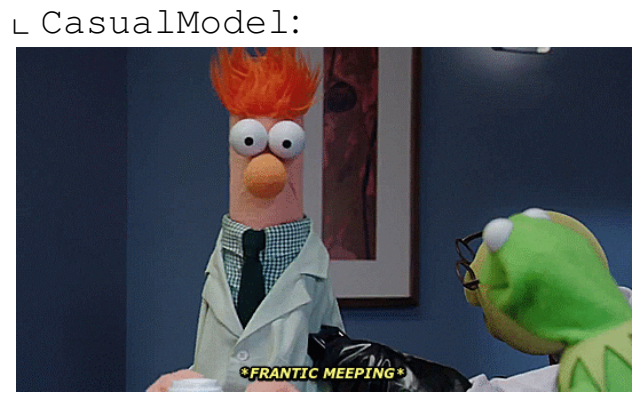

Figure 1: Gif responses in conversation like the one shown above are embodied dialog that use visual imagery to convey reactions and emotions. This paper develops a system to select the appropriate gif response to messages. (PDF best viewed with Adobe Acrobat)

Conversation analysis is central to NLP and multiple approaches have analyzed this dialog structure (Jurafsky et al., 1998; Pareti and Lando, 2018; Cohn et al., 2019) and developed conversational agents to engage with people (e.g., Fang et al., 2018; Xu et al., 2020; Hong et al., 2020). Recent work has focused on generating open domain social chatbots that engage in sustained conversations in a natural way (Ram et al., 2018). Because many of these systems are designed to support voicebased dialog, they overlook non-textual forms of interaction used in social media conversations. In parallel, multimodal NLP systems have been developed for image data, often focusing on image-totext tasks such as image captioning (Melas-Kyriazi et al., 2018; Sharma et al., 2018) and visual question answering (Antol et al., 2015; Huang et al., 2019; Khademi, 2020). More recent work has focused on the reverse text-to-image dimension, such as generating an image from a description (Niu et al., 2020; Ramesh et al., 2021). Our work unites these two strands of research by integrating imagebased communication into conversational agents.

Our paper offers three main contributions. First, 
we propose the new task of selecting gif responses in multimodal conversation analysis and introduce a new dataset of 1,562,701 real-world conversation turns with gif replies. Second, we introduce a new model PEPE THE KING PRAWN that fuses image and text-based features to select a relevant gif response. In in-house experiments, we show that our model substantially outperforms strong baseline models at selecting the exact gif used in real data and, in a manual test of the quality of the best responses, achieves an nDCG of 0.8145 on the annotated test set. Third, in a real-world test, we deploy our model as a part of a large-scale randomized controlled trial and show that the gif replies produced by our model are more highly voted by the community. Data, code, and models are available at https://github.com/xingyaoww/gif-reply.

\section{GIF Communications}

Gifs have been widely adopted in communication as a natural form of embodied speech where the visual imagery conveys emotions or a reaction as a response (Bakhshi et al., 2016; Tolins and Samermit, 2016). These gifs commonly come from widelyknown cultural products, such as movies or television shows, which provides common knowledge for how they could be interpreted (Eppink, 2014; Miltner and Highfield, 2017). However, a single gif may have multiple interpretations, depending on the context, cultural knowledge of its content, and the viewer (Jiang et al., 2017). As a result, a single gif can serve multiple functions in communication (Tolins and Samermit, 2016).

Gifs have grown in their use through increasing affordances by platforms like Tumblr, Reddit, Imgur, and Twitter that allow gifs to be natively displayed like text in conversation threads (Jiang et al., 2018). Further, gif-based keyboards have been introduced that allow users to search for gifs that have been tagged with keywords or other metadata (Griggio et al., 2019). Yet, these technologies require that gif data be prepared with sufficient tags to be searchable or to have sufficient data to use collaborative filtering techniques for recommendations (Jiang et al., 2018, p.9). As a result, there is a clear gap in identifying appropriate response gifs directly from the text, which this work fills.

\section{Data}

Despite the widespread use of gifs, no standard dataset exists for text and gif replies. Further, al- though platforms like Twitter support gif replies, these gifs are not canonicalized to identify which responses correspond to the same gif. Therefore, we construct a new dataset for this task by collecting responses, matching their images, and augmenting this data with metadata about the gif, where possible. A visual description of the whole procedure can be found in Appendix Figure 7.

\subsection{Gif Response Data}

Gifs have many uses (Miltner and Highfield, 2017) and so we use a two-step approach to collect data that focus specifically on those likely to be used in conversation. First, gif responses are collected from Twitter by identifying all replies to Englishlanguage tweets containing animated_gif as embedded media. Tweets were collected from a $\sim 10 \%$ sample of Twitter from March 13th, 2019 to Jan 24th, 2020, totaling 42,096,566 tweets with a gif that we were able to retrieve. Twitter does not canonicalize its gifs so two separate gif files may actually have the same imagery. Further, these files may not be identical due to small differences such as color variations or aspect ratios. To identify uses of the reference gifs, we use Average Hash from the imagehash library to create low-dimensional representations of each gif where hash distance corresponds to perceptual distance. Since gifs are animated and may contain varying scenes, we compute the hash for the first, middle, and final frames, concatenating these into a single hash. Two gifs are considered the same if (i) they have identical hashes or (ii) their hamming distance is $<10$ and gifs with that hash have been used more than 500 times in Twitter. This latter condition was selected after manual evaluation of thresholds to trade-off between increasing the size of the training data and reducing potential noise caused by matching error. A visual example of this process can be found in Appendix Figure 8.

Not all gif responses in the Twitter data are conversational or appropriate for wider re-use. Therefore, we filter these responses to only those gifs whose imagery matches gifs hosted by the Giphy website, which is the backend for many gif-based keyboards. Giphy contains a wide collection of gifs that are curated to remove content inappropriate for general use (e.g., violent or sexual imagery). Gifs on the platform are categorized (e.g., "reaction" or "celebrities") and we identify 28 categories containing 972 keywords likely to contain gifs used 


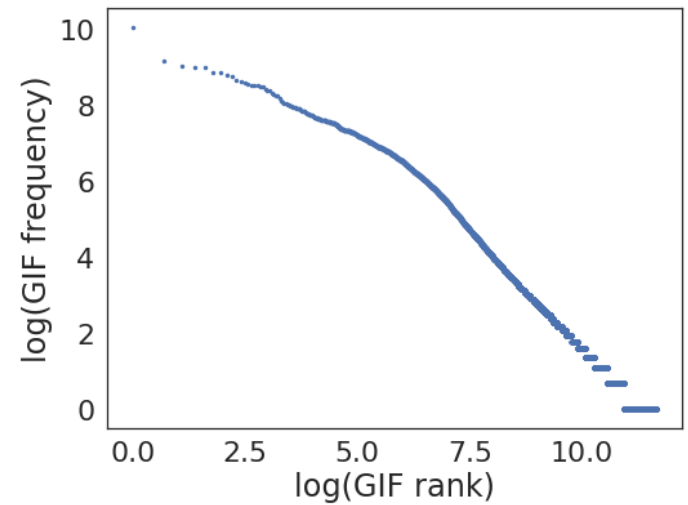

Figure 2: The frequency distribution of gifs in our data roughly follows a log-normal distribution, with a few gifs used often, while a long tail of gifs are used relatively infrequently.

in conversation. A total of 2,095,993 gifs linked to those keywords were ultimately retrieved and stored as image hashes. Additional details of categories and keywords are in Appendix B.

After the matching image hashes to filter replies, we identify 115,586 unique gifs, referred to as reference gifs, and 1,562,701 tweet replies using one of these gifs, which forms our official dataset. Figure 2 shows these gifs' frequency in the data; much like words, a handful of gifs receive widespread use, while a long tail of gifs are rarely used.

\subsection{Gif Metadata}

We augment our gif data with information about their content. Some gifs have text that transcribes what a person is saying in the gif's scene or is a meta-commentary on the content. This text is extracted using paddleOCR (Du et al., 2020). Since some gifs are long enough to contain multiple utterances, we run OCR on four frames sampled from each quartile of the gif's length. Roughly $50 \%$ $(58,020)$ of gifs contain at least one extracted word from the selected frames, with an mean of $5.5 \mathrm{ex}-$ tracted words per gif across the dataset.

Second, some gif repositories like Giphy allow users to tag gifs with information on their content or theme, e.g., "face palm" or "movie." We collect tags for the $115 \mathrm{~K}$ reference gifs used in Twitter, obtaining 39,651 unique tags. These user-generated tags were moderately noisy due to orthographic variations like spelling, capitalization, and spacing. Therefore, we merge tags by (i) lower-casing the text and (ii) performing a manual merge for similar word forms (e.g., "excited" and "exciting"). To minimize noise, we retain only tags that have been used with at least five gifs and where those gifs have been used at least 1000 times in total; this process removes many low-frequency tags that are either overly-specific or idiosyncratic in their use.

Finally, we performed a manual inspection of all remaining tags to remove tags that are too general (e.g., "emotion") and retain only noun, adjective, and verb tags (words or multi-word expressions) that describe specific emotions or actions. A total of 241 unique tags were retained (Appendix C). $6.0 \%$ of gifs have at least one tag associated with them (mean 1.9 tags). However, these tagged gifs account for $38.7 \%$ of the replies in our dataset, suggesting tags are only available for more-popular gifs. Our dataset represents roughly an order of magnitude more data and more tags than the closest related dataset of Chen et al. (2017) that contained $23 \mathrm{~K}$ gifs with 17 manually-curated emotions.

\section{Gif Reply Models}

We introduce a series of models for producing a gif response in conversation. Each model will select a gif from the $115 \mathrm{~K}$ gifs in our dataset as a response to a text-based message. This task is related to but distinct from work on image-text matching (Lee et al., 2018), which aims to find an image describing a piece of text, or text-to-image (e.g., Wen et al., 2015; Xu et al., 2018), which generates an image from a text description. Here, we aim to select gifs that reflect natural continuations or reactions to a message in a dialog, akin to how gifs are used in social media. For all models, additional details on the training procedures and hyperparameters are provided in Appendix A. The three models that follow use varying degrees of information about the gifs and text to select a response.

\subsection{Tag-based Predictions}

The first model uses tags as a shared representation for characterizing gifs and text. Analogous to how object tags are used as anchor points for image-text matching (Li et al., 2020) and pivot languages are used in machine translation (Cheng et al., 2017), we use tags to bridge information between the text in a tweet and the visual content of a gif. Here, each gif becomes associated with a set of tags describing its conversational functions and for each text, we predict the set of tags for gifs responses to it - in essence, predicting what types of responses are most appropriate. We describe both of these processes next and how gifs are ultimately selected. 
Estimating Gif Tags Only 6.0\% of the gifs in our data have associated tags. Therefore we train a neural model to predict tags using known tags as training data. To capture any changes in emotion or imagery across the gif, we make separate predictions for four frames sampled across the gif (the same used in \$3.2). Each frame is passed through an EfficientNet-based (Tan and Le, 2019) GIF encoder, shown in Figure 3, to extract a lowdimensional feature vector from each frame. These frame embeddings are fused using the attention mechanism from a transformer encoder layer. The output of the transformer feeds into a fully connected layer, which is trained as a multi-label classifier using binary cross-entropy to predict which tags should be present.

Predicting Response Tags for Text For each message, we predict the $k$-hot distribution of tags for a gif response by training a BERTweet model (Nguyen et al., 2020), which has been pre-trained on a large corpus of Twitter data (shown as "Tweet Encoder" in Figure 3). The model with an additional fully connected layer is trained as a multilabel classifier using binary cross-entropy, using the tags for the gifs used in reply (if known).

Tag-based Gif Selection At inference time, given a message, we use the text-to-tag model to predict a $k$-hot distribution over tags. Then, we select the gif whose estimated tag distribution is closest in Euclidean distance.

\subsection{CLIP variant}

The second model uses an end-to-end training approach based on the architecture of OpenAI CLIP (Radford et al., 2021). The architecture features two encoders, one for text and one for images. During training, the encoders are updated using contrastive loss that maximizes the cosine similarity of paired image-text representations and minimizes the cosine similarity of random pairs of images and texts. We replicate the CLIP architecture and training procedure, using BERTweet to encode text and EfficientNet (Tan and Le, 2019) to encode a composite image of four frames from the gif (compared with BERT and ResNet in their implementation). While originally designed to select an image for a text description, our model is trained to select a gif reply for a text message - a more challenging task than the image retrieval task used in the original CLIP setup, as the message may not contain words describing elements of the gif. At inference time, given a tweet, we use the trained tweet encoder to extract its representation and compute its cosine similarity with each encoded representation for our gifs. The gif with the highest cosine similarity is returned as the best response.

\subsection{Pepe the King Prawn}

Our final model, KING PRAWN ${ }^{1}$ (referred to as "PEPE".) selects gif responses by using a richer set of multimodal features to create a gif representation. Rather than encode the gif solely from its image content, we use a multimodal encoder that captures (i) any text it might have, (ii) the types of objects present in the gif, and (iii) object regions as visual features. We encode these gif aspects using an OSCAR transformer (Li et al., 2020) to create a unified representation, shown in Figure 3 (bottom). Object names and regions of interest feature vectors are extracted using a pre-trained bottom-up attention model (Anderson et al., 2018).

As input to the OSCAR encoder, the captions to each of the gif's four frames are concatenated together with an "[INTER_FRAME_SEP]" separator token. We filter object areas detected by the bottomup attention model (Anderson et al., 2018) and we keep all objects with probability $>0.5$. We then concatenate object names together with the same inter-frame separator between names of different frames. Together, the caption text, object names, and image-region features are fed into the OSCAR transformer encoder to generate a GIF feature vector; the transformer is initialized with the default OSCAR weights. We use BERTweet to encode text. The entire PEPE model is trained end-to-end using contrastive loss, similar to the CLIP model.

\section{Evaluation}

We initially evaluate the methods in two ways. First, we use traditional classification-based evaluation, testing whether the models can reproduce the observed gif replies. However, some messages could have multiple valid gif responses. Therefore, as a second test, we evaluate the model in a retrieval setting, measuring whether its most-probable responses are good quality for a message.

Experimental Setup Models are trained and tested on a dataset containing 1,562,701 Tweet-

\footnotetext{
${ }^{1}$ KING PRAWN refers to "selecKting INteresting Gifs for Personal RespAWNses." In this crazy muppet-name-landgrab world we live in, our only regret is that we couldn't get "Pepino Rodrigo Serrano Gonzales" to fit as a bacronym, which we leave to future work.
} 


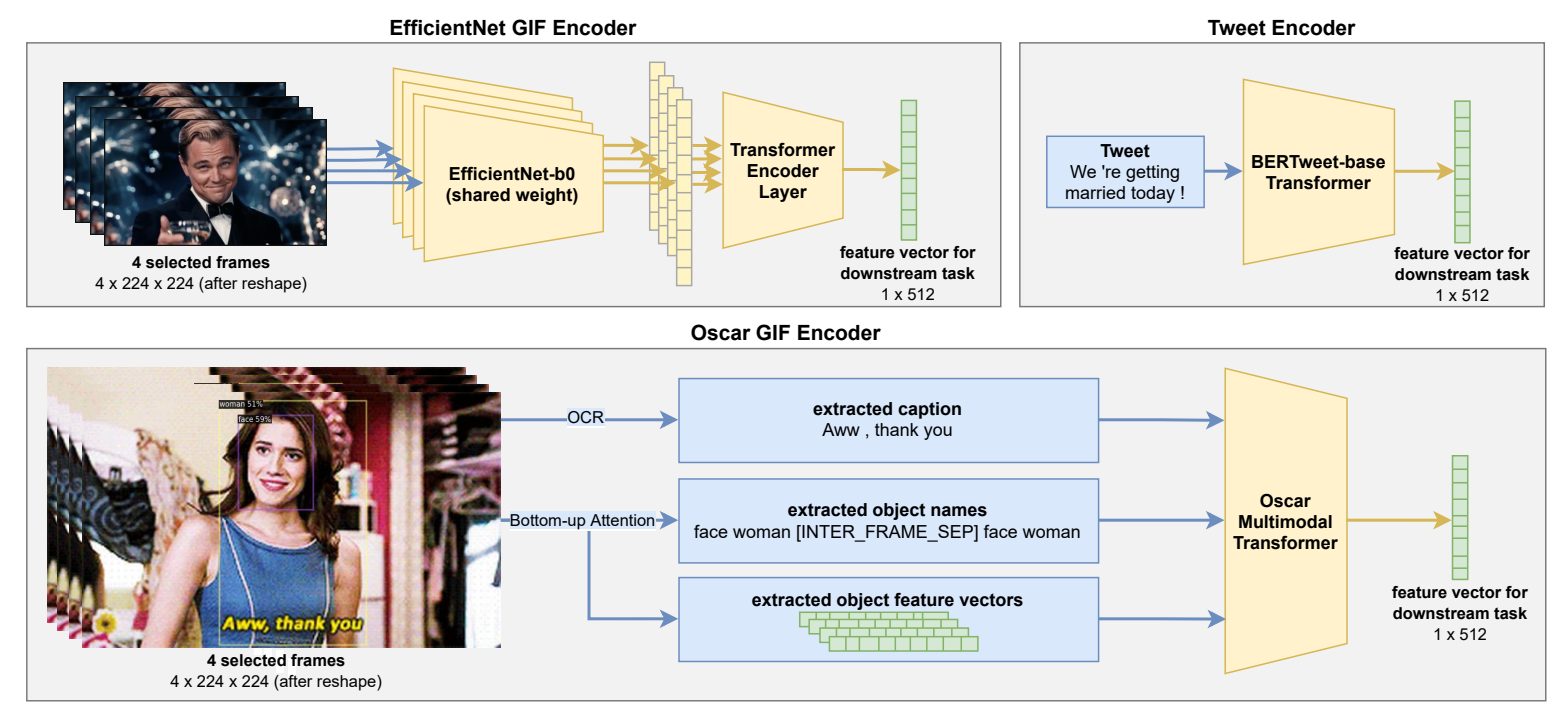

Figure 3: The different encoder modules used to construct the models in $\S 4$.

GIF pairs associated with 115,586 unique gifs, where 605,063 tweet-gif pairs are associated with at least one tag. Using the finalized 241 unique tags as classes for multi-label classification, we split the dataset by stratify on tags using the iterative train-test split method provided by scikit-multilearn library (Sechidis et al., 2011; Szymański and Kajdanowicz, 2017) to create a 80:10:10 train, dev, and test split which is finalized to train the models described in $\S 4$. Following BERTweet (Nguyen et al., 2020), we preprocess tweets in our dataset using NLTK TweetTokenizer for tokenization, emoji package to translate emotion icons, and converted mentions and links to special "@USER" and "HTTPURL" tokens.

Annotated Data To test whether each model's predictions are valid responses, we annotate the ten most-probable gif predictions for a subset of the tweets in our test data. Many tweets in our test set require substantial context to understand due to having few tokens, linking to URLs that provide extra knowledge, mentioning other users in directed communication. These factors suggest social context or general knowledge aids in the recipient's understanding of the gif's intentions. While the model can still benefit from training on such examples, judging the appropriateness of response is difficult without access to the social context. Therefore, to reduce interpretation ambiguity, we annotate only tweets without URLs or user mentions and having at least 10 tokens. This process selects tweets with sufficient content to judge appropriateness independent of the larger social context.

Two annotators (the authors) were shown a list of potential gif responses for a tweet and asked to judge whether this is an appropriate gif response (a binary rating). Gifs were selected from the ten most-probable replies for each system and collectively shown in random order to prevent knowing which system generated each reply. A total of 2,500 gif-tweet pairings were annotated. Annotators attained a Krippendorf's $\alpha$ of 0.462; while moderate agreement, this value is expected given known differences in how people interpret and value gif responses based on their familiarity with its content, message interpretation, and life-experience (Jiang et al., 2018). We follow the evaluation setup from other retrieval-based dialog systems (e.g. Yu et al., 2021; Kumar and Callan, 2020) and use normalized Discounted Cumulative Gain (nDCG), which measures whether more appropriate gif responses are ranked higher. A gif's appropriateness score is the sum of annotators' ratings.

Results The PEPE model was able to identify relevant and good-quality gif responses, as shown by its performances on the test data (Table 1) and annotated data (Table 2). Performance on the test set is expected to be low, given the challenge of identifying the exact gif used for a tweet when multiple possible gifs are likely to be equally valid. However, the PEPE model is still able to identify the exact gif (out of $115 \mathrm{~K}$ ) in its top 10 predictions for $3 \%$ of the data, substantially outperforming all 


\begin{tabular}{lrrr} 
Model & Top-1 & Top-5 & Top-10 \\
\hline Tag-based & 0.000000 & 0.000092 & 0.000119 \\
Random & 0.000020 & 0.000059 & 0.000158 \\
CLIP variant & 0.000488 & 0.001669 & 0.002783 \\
Distribution sampling & 0.000996 & 0.005098 & 0.009780 \\
PEPE & $\mathbf{0 . 0 0 5 3 7 5}$ & $\mathbf{0 . 0 1 8 7 2 3}$ & $\mathbf{0 . 0 3 0 9 1 8}$
\end{tabular}

Table 1: Models' precision-at- $k$ on selecting the exact gif used as a response for a tweet in the test set; this performance is an underestimate of each model, as many model-predicted gifs may be appropriate.

\begin{tabular}{ll} 
Model & nDCG \\
\hline Random & 0.3273 \\
Tag-based & 0.4526 \\
Distribution sampling & 0.4969 \\
CLIP variant & 0.5934 \\
PEPE & $\mathbf{0 . 8 1 4 5}$
\end{tabular}

Table 2: Models' nDCG scores at proposing appropriate gif replies, measured from annotations on the top 10 most probable gif replies of each model.

other models.

Performance on the annotated data (Table 2) provides a more realistic assessment of whether models can generate high-quality replies, as it measures whether the models' replies themselves were good. The PEPE model attains substantially higher performance $(p<0.01)$ than other models. While the CLIP variant model performs well, the contentagnostic Distribution sampling baseline performs nearly as well. This baseline's high performance speaks to the multiple interpretations of gifs and the ease at which readers can make connections between a gif and message. Indeed, even the randomgif model has a non-zero nDCG, highlighting the ability for an arbitrary gif to still be considered appropriate. We speculate that popular gifs may be popular because of this ease of multiple interpretations. Table 4 shows the top predictions for models and baselines for two example messages, illustrating the variety of relevant gifs; the PEPE and random baseline replies for the second message exemplify the type of gifs that can be widely applied to many messages, often to humorous effects.

Ablation study PEPE fuses multiple types of input, which may uniquely contribute to model's ability to select gif replies. To understand how these inputs each contribute, we performed an ablation study on the annotated test set by removing one input from Oscar GIF Encoder shown in Figure 3 (i.e., a gif's caption, object names, or objects' visual features)

\begin{tabular}{ll} 
Model & nDCG \\
\hline PEPE & $\mathbf{0 . 8 1 4 5}$ \\
PEPE without object names & 0.7665 \\
PEPE without caption & 0.7559 \\
PEPE without object features & 0.7533
\end{tabular}

Table 3: Results for ablated versions of PEPE where specific input is removed (cf. Table 2) show that all input forms contribute to the ability to select replies.

and evaluating the model's resulting gifs on the same test instances.

The ablated model performances, shown in Table 3, reveal that each input is useful for selecting gifs. $^{2}$ Object features capture visual information about what specifically is present in the gif (beyond the discrete names of what is present, e.g., "person" or "building") and show that multimodality is important for high performance-predicting replies just from a gif's caption and categorized content are insufficient. Similarly, the caption of a gif (if present) is important, as the text can help make explicit the intended interpretation of a gif.

\section{Field Experiment}

To test the generalizability of our models and quality of their responses, we conduct a large-scale randomized controlled trial (RCT) that has the models respond to real users and measure their perception of reply quality. ${ }^{3}$

\subsection{Experimental Setup}

Gifs were posted to the Imgur platform, which is a highly active social media community that supports both image and text-based interactions. On Imgur, users may create posts, which contain one or more images with optional commentary, or comment on posts or replies. Similar to pre-2018 Twitter, comments are limited to 140 characters. Imgur conversations are threaded and frequently contain both image and text comments. Like Reddit, users may upvote and downvote content, providing a score of how well it was received by the community; we use

\footnotetext{
${ }^{2}$ The performance decrease for removing object names is statistically significant ( $\mathrm{p}<0.01$, bootstrapped). The decreases for removing captions and objects' visual features are significant from the name-removal model $(\mathrm{p}<0.01)$ but the two models are statistically equivalent $(\mathrm{p}>0.19)$.

${ }^{3}$ This experiment was ruled as Not Regulated by the University of Michigan IRB (HUM00197631). However, IRB approval is not sufficient to prevent harm (Bernstein et al., 2021) and significant precautions were taken to minimize potential risk (See \$9) .
} 


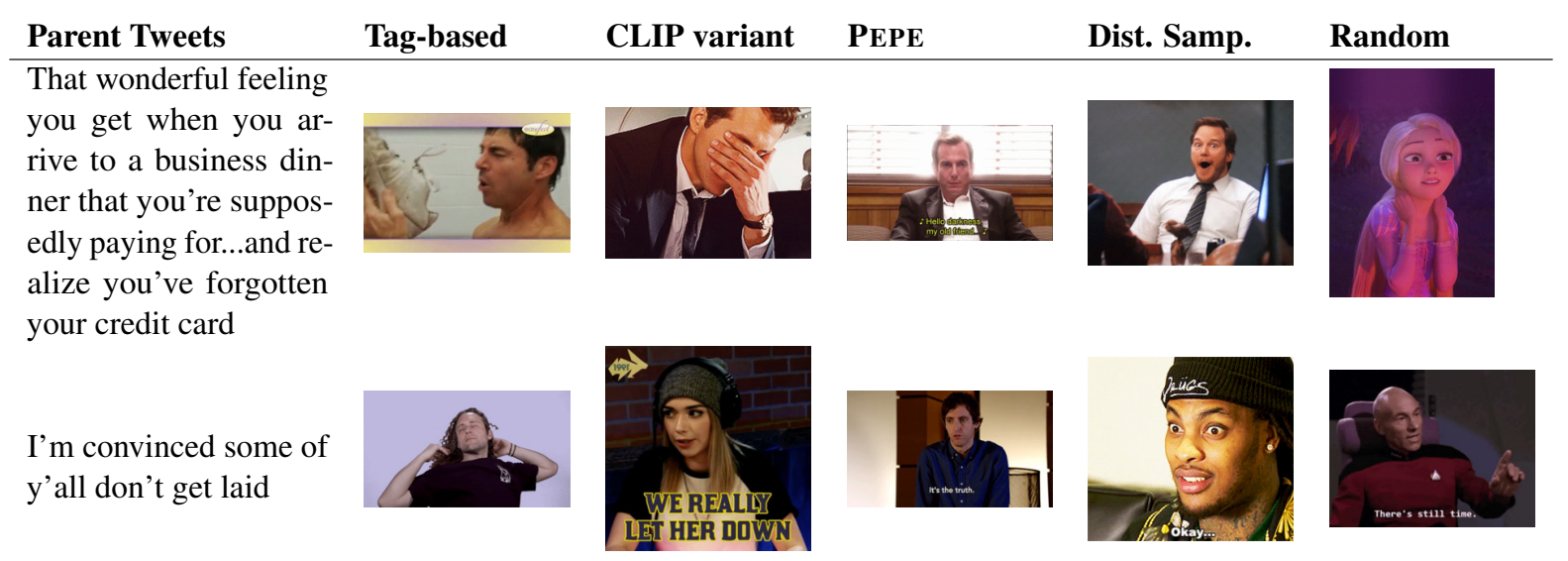

Table 4: Model-selected replies to messages (paraphrased for privacy). Click an image to view the gif on Giphy.

this score in our experiments to evaluate quality.

Our experiment focuses on generating Gif-based replies to top-level text comments (comments made directly to the post). This setup mirrors the conversational data our models were trained on. Imgur supports several ways of filtering its stream of posts. To ensure that our replies have sufficient visibility, we select posts that have already receive $10 \mathrm{com}$ ments and appear in the "most viral" sorting. From these posts, we reply to the top-rated text comment. The RCT runs from 8 AM to 8 PM (local time), making at most 10 replies per hour.

Not all topics or comments are suitable for automated responses and great care was taken to prevent potential harm to the community. Through multiple rounds of testing which replies would be responded to, we curated a list of keywords that could lead to potential controversial replies, such as terms about religion or race (full list in Appendix D). Any comment containing a token or lemma matching a word on this list is excluded and not replied to. As a further safeguard, experimenters monitored all replies to remove any that were deemed inappropriate. See the Ethics Section (§9) for a longer discussion of safeguards.

The field experiment consists of five arms, corresponding to the three trained models and the two baseline models. During each trial, one model is selected and generates a response; the trained model replies with the most probable gif. ${ }^{4}$

Not all models are equally likely to perform well and so to make the most use of our trial budget,

\footnotetext{
${ }^{4}$ Due to a bug, early experimental trials for the CLIP and PEPE models used the tenth most-probable gif; however, using the ratings in the annotated data, a $t$-test of the difference in quality for most- and tenth-most probable gifs showed no statistically-significant difference in quality for both models $(p>0.1)$. Therefore, we include this data in our results.
}

we use Thompson sampling (Russo et al., 2018) to randomly select which arm of the trial to use. Thompson sampling builds a probability model for the estimated reward of each arm (here, the score a reply receives) and samples from the model such that higher-rewarding arms are sampled more frequently. As a result, this method can provide tighter estimates for the reward of the most useful arms. Scores in Imgur have a skewed distribution, with few comments receiving very high scores and most receiving near the default score (1). Therefore, we use Poisson Thompson sampling. Some comments may be downvoted to receive scores below zero, so for simplicity, we truncate these scores to 0 .

We initialize the reward estimates for our experiment by selecting one of the five models in a round-robin manner to reply to an Imgur comment for 3 days. These initial scores act as priors for Thompson sampling to update Poisson distributions for each model. In the trial, we choose a model by sampling from the up distributions using all previous days' scores as the prior. The experiment ran from April 15th, 2021 to August 30th, 2021 , and models generated a total of 8,369 replies.

To evaluate the results of the RCT, we construct a Negative Binomial regression on the dependent variable of the score received for a model's reply, truncating negative scores to zero. The Negative binomial was chosen instead of Poisson due to over-dispersion in the score variable. The models are treated as a categorical variable, using the random model as a reference. Since the score will depend, in part, on the attention received by the parent post and comment (higher-rated comments are displayed first), we include linear effects for the post and parent comment. Finally, we include five text-related variables to control for the con- 


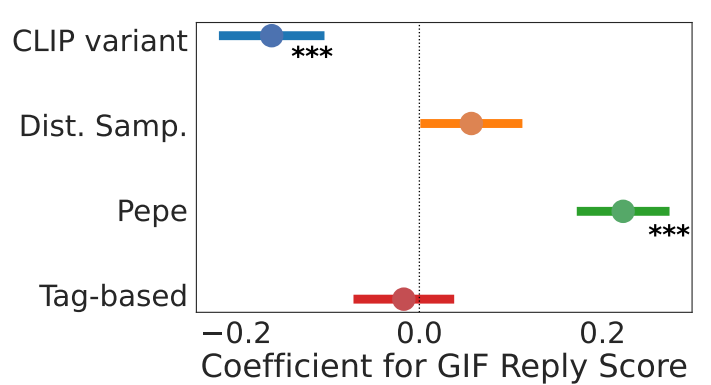

Figure 4: Negative Binomial regression coefficients for each model on predicting a gif reply's score, using the random-gif model as the reference category; bars show standard error and $* * *$ denotes significance at 0.01 .

tent of the parent comment: the topic distribution (Appendix Table 9) from a 10-topic model (dropping one topic due to collinearity), the sentiment and subjectivity of the message estimated using TextBlob library, the length of the comment, and whether the comment contained a question.

\subsection{Results}

The field experiment demonstrates that the PEPE model is able to generate significantly higherscoring responses. Figure 4 shows the Negative Binomial regression coefficients for the three models and empirical distribution baseline, with the random gif model as a reference; full regression results are shown in Appendix Table 6. The PEPE model substantially outperforms all other models $(\mathrm{p}<0.01)$ in this real-world setting. Surprisingly, despite performing second-best in our annotated evaluations, the CLIP model performs worst, with its replies receiving fewer upvotes than the two baselines that randomly select gifs. We investigate potential explanations for these performances next.

The Random and Distributional-sampling baseline models perform surprisingly well relative to models that take the text and gif content into account, with only the PEPE model outperforming them. The performance of the random baselines matches prior work showing people are still able to draw some connection between their interpretation and the reply (Madden, 2018, p.29). Further, we observed that, when the model's reply truly seemed random, some users replied say they upvoted solely because they enjoyed the gif.

As a follow-up experiment, we tested whether models could be getting higher (or lower) scores by repeatedly picking the same gifs that are skewed towards a positive or negative reaction. Figure 5 shows the score distribution for the top ten most fre-

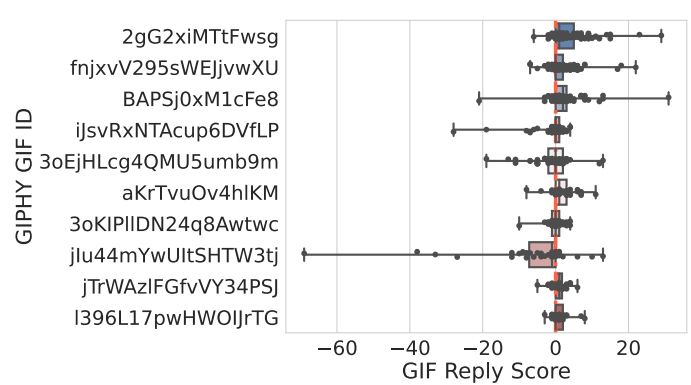

(a) Tag-based

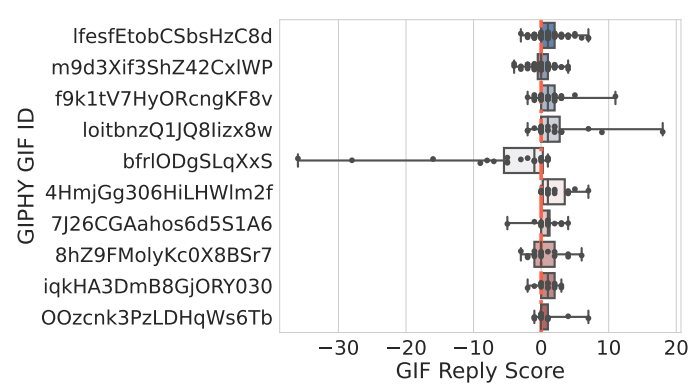

(b) CLIP variant

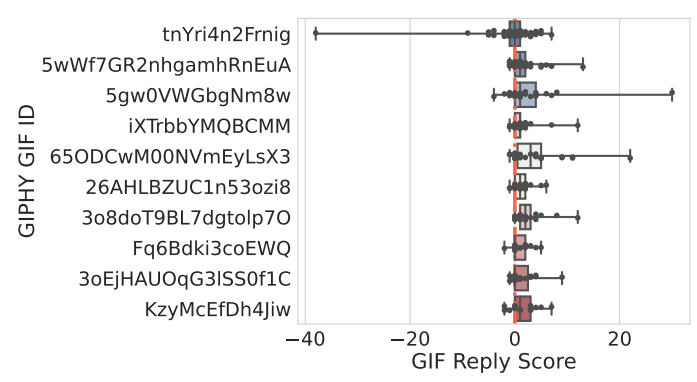

(c) PEPE

Figure 5: Score distributions for most-frequently used gifs show few are universally skewed positive. Boxes show quartile ranges; gifs are in Appendix Table 7.

quently used gifs (visual examples in Appendix Table 7) for each of the three trained models and reveals surprisingly divergent behavior for how the community reacts. Each model had a different set of most-used gifs, indicating the models did not converge to a universal set of common replies. Indeed, a gif's frequency-of-use and mean reply score were uncorrelated in all three models $(r \approx$ $0.01, \mathrm{p}>0.73$ for all models). The most-used gifs for each model had average scores that were positive, but the distributions for each gif show that some uses were occasionally downvoted. This high variance in scores indicates that a gif's intrinsic qualities are not solely responsible for the received score and, instead, appropriate use in context is plays a significant part in community reception.

We examined whether models relied on the same set of gifs. Figure 6 shows the distribution of gif 


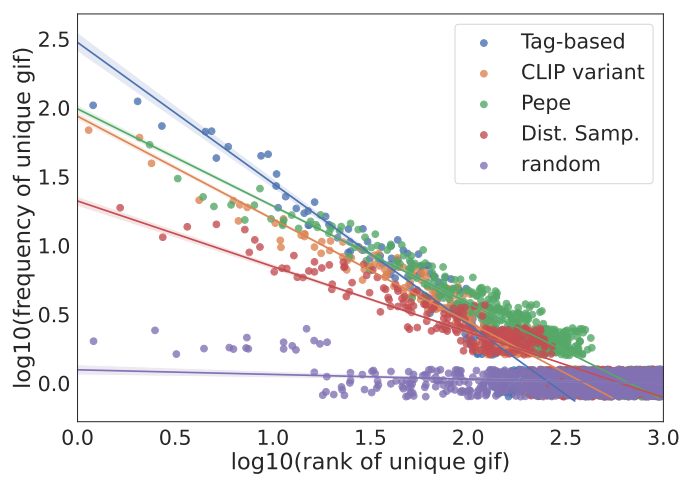

Figure 6: Gif use frequency by each model, shown as frequency-vs-rank log-scaled with first-order line fit (jitter added for separation).

uses by each model, indicating that the tag-based model relied frequently on a small set of gifs. However, the PEPE and CLIP variant models were substantially more varied, indicating they draw from the long-tail of possible gifs.

Do any of our models spark more subsequent conversation? We fit a separate Negative Binomial regression on the total number of comments made to our reply, using the same IVs as the score regression and include the reply's score itself as another IV. This model (Appendix Table 8) shows that both the distributional-sampling baseline and PEPE models produced replies that led to fewer subsequent comments $(\mathrm{p}<0.01)$-despite the PEPE model having the most-upvoted replies. However, the score of the gif reply was positively associated $(\mathrm{p}<0.01)$ indicating that more appropriate replies do receive more subsequent conversation. We speculate that the random models may have led to more conversation due to users replying to express confusion about why the particular gif was used. This result points to a need to understand what text and visual factors in gifs influence the volume of subsequent dialog and an opportunity to optimize gif models for both quality and number of conversation turns.

\section{Related Work}

This work draws upon two strands of research from dialog systems and multimodal NLP. Conversational dialog systems have traditionally been built upon large-scale dialog corpora from social media platforms (Bessho et al., 2012) such as Twitter. Our approaches are fundamentally information retrieval based systems that mirror the approach by text-based conversational systems that retrieve ex- isting messages from a large social media corpus as potential replies and rank these to select a response. Our work mirrors models that use neural networks for ranking (Yan et al., 2016; Inaba and Takahashi, 2016; Penha and Hauff, 2021, e.g.,); however, we note that many recent knowledge-grounded and open domain models use encoder-decoder methods to improve versatility and applicability (e.g., Ghazvininejad et al., 2018; Gao et al., 2019; Zhou et al., 2020). Generative approaches are likely inappropriate for gif-based conversation as gifs are more akin to mimetic artifacts that build on cultural knowledge (Eppink, 2014), making synthesizing a new gif from scratch likely less effective.

All three models used here rely on joint embedding spaces for gif and text. Multiple works in NLP have been proposed to align these representations (Kiros et al., 2014; Wang et al., 2016), often for particular applications such as visual question answering (Antol et al., 2015). Recent work has focused on embeddings these media with a single encoder that takes both text and images as input (e.g., Wang et al., 2019; Chen et al., 2020), in contrast to our model that uses separate image and text encoders (Figure 3); these multimodal encoders are prohibitively computationally expensive to use in our setting during inference time, as the model would need to be run on each gif (and message) to rank replies, compared with our model that only needs to encode text. However, performance and efficiency improvements in aligning image and text representations would likely benefit our task.

\section{Conclusion}

People like using gifs in online conversations-gifs are a fun and playful way to communicate. However, modern NLP conversational agents operate only by text. Here, we introduce a new dataset of $1.56 \mathrm{M}$ conversation turns using gifs, including captions and metadata, and develop a new conversational model PEPE THE KING PRAWN that selects appropriate gif responses for messages through comparing encoded gif and text representations. In two evaluations, we show that PEPE is able to generate highly-relevant gif responses and in a largescale RCT, we show that the gif replies from the PEPE model received significantly higher scores from the general public. Our work demonstrates the opportunity for using NLP methods to successfully engage in multimodal conversations. 


\section{Ethics}

The interactive nature of the RCT necessitated a close consideration of ethical issues (Thieltges et al., 2016). Prior to beginning the RCT, the study team obtained IRB approval to interact with users. While necessary in the legal sense, IRB approval is not sufficient to justify the ethical grounds of the study. The primary risks of the study are if the automated models respond with an inappropriate gif or respond to a message that is not suitable for automated response (e.g., discussing the death of a loved one or making an offensive statement). These risks were mitigated in multiple ways throughout the dataset construction and field experiment.

First, the selection criteria for which comments we reply to was designed to only reply to content that was already deemed appropriate by the community. By selecting only posts that had received sufficient upvotes to be called "viral" and were already receiving comments, we mitigate the risk of engaging in topics or conversations that are inappropriate according to the norms of the Imgur community, as these posts would be removed by moderators or would have received sufficient downvotes to stay in obscurity.

Second, by focusing on the top-voted comment to these posts, we again reply to content that has already been deemed high-quality by the comment. This comment-level criteria substantially lowers the risk of our models commenting on inappropriate comments (e.g., a comment insulting another user), as these comments are readily downvoted by the community prior to our intervention.

Third, we employed extensive filtering to avoid replying to any comment containing a potentially sensitive topic, e.g., a discussion of race or trauma (keywords are listed in Appendix D). The initial set of keywords was developed through examining potentially sensitive topics and then iteratively added to by simulating which messages our RCT would reply to and examining whether it would be appropriate. During the field RCT, experimenters continuously monitored the comments to ensure no harm was being done. Ultimately, only three comments were removed during the initial two days, which was due to a bug in the lemmatization and these comments should have been filtered out by our earlier criteria; these comments were removed quickly and we did not observe any notable response from the community.

Fourth, one risk is replying with an inappropri- ate gif, which is mitigated by the use of Giphy to seed our initial gifs. As this platform is curated and does not host objectively offensive gifs (e.g., overly-violent content), our initial gif set is relatively free of objectionable gifs. Because our model learns directly from gifs' frequency of use, unless objectively offensive gifs are widely used, they are unlikely to be deployed from our RCT; we speculate that few objectively offensive gifs are widely used and, in practice, we have not identified any during the study period or when examining hundreds of random gifs in our data (or used in the RCT).

Finally, one risk is that by learning gif responses from observed data, our models may reinforce cultural stereotypes that are encoded in the gifs themselves (Erinn, 2019), e.g., the association of African American individuals with strong emotions. While our gif data is relatively clean of overtly offensive gifs, we acknowledge that our model likely does inadvertently perpetuate some of these latent biases in the data. However, the success of our model suggests a future mitigation strategy for platforms suggesting gifs: as biases become known, our approach can be used to suggest less-biased gifs as potential responses to mitigate future harm.

\section{Acknowledgments}

We thank the reviewers, area chairs, and senior area chairs for their thoughtful comments and feedback. We also thank the Blablablab for helpful feedback and letting us deploy PEPE to the group's Slack and putting up with the ridiculous (and hilarious) gif replies and Imgur for being a wonderful community. This material is based upon work supported by the National Science Foundation under Grant No. 2007251.

\section{References}

Peter Anderson, Xiaodong He, Chris Buehler, Damien Teney, Mark Johnson, Stephen Gould, and Lei Zhang. 2018. Bottom-up and top-down attention for image captioning and visual question answering. In 2018 IEEE Conference on Computer Vision and Pattern Recognition, CVPR 2018, Salt Lake City, UT, USA, June 18-22, 2018, pages 6077-6086. IEEE Computer Society.

Stanislaw Antol, Aishwarya Agrawal, Jiasen Lu, Margaret Mitchell, Dhruv Batra, C. Lawrence Zitnick, and Devi Parikh. 2015. VQA: visual question answering. In 2015 IEEE International Conference on 
Computer Vision, ICCV 2015, Santiago, Chile, December 7-13, 2015, pages 2425-2433. IEEE Computer Society.

Saeideh Bakhshi, David A. Shamma, Lyndon Kennedy, Yale Song, Paloma de Juan, and Joseph Jofish Kaye. 2016. Fast, cheap, and good: Why animated gifs engage us. In Proceedings of the 2016 CHI Conference on Human Factors in Computing Systems, San Jose, CA, USA, May 7-12, 2016, pages 575-586. ACM.

Michael S Bernstein, Margaret Levi, David Magnus, Betsy Rajala, Debra Satz, and Charla Waeiss. 2021. Esr: Ethics and society review of artificial intelligence research. ArXiv preprint, abs/2106.11521.

Fumihiro Bessho, Tatsuya Harada, and Yasuo $\mathrm{Ku}-$ niyoshi. 2012. Dialog system using real-time crowdsourcing and Twitter large-scale corpus. In Proceedings of the 13th Annual Meeting of the Special Interest Group on Discourse and Dialogue, pages 227231, Seoul, South Korea. Association for Computational Linguistics.

Elli Bourlai and Susan C Herring. 2014. Multimodal communication on tumblr: "i have so many feels!" In Proceedings of the 2014 ACM conference on Web science, pages 171-175.

Weixuan Chen, Ognjen Oggi Rudovic, and Rosalind W Picard. 2017. Gifgift: Collecting emotional animated gifs with clustered multi-task learning. In 2017 Seventh International Conference on Affective Computing and Intelligent Interaction (ACII), pages 510-517. IEEE.

Yen-Chun Chen, Linjie Li, Licheng Yu, Ahmed El Kholy, Faisal Ahmed, Zhe Gan, Yu Cheng, and Jingjing Liu. 2020. UNITER: Universal ImageTExt representation learning. In ECCV.

Yong Cheng, Qian Yang, Yang Liu, Maosong Sun, and Wei Xu. 2017. Joint training for pivot-based neural machine translation. In Proceedings of the TwentySixth International Joint Conference on Artificial Intelligence, IJCAI 2017, Melbourne, Australia, August 19-25, 2017, pages 3974-3980. ijcai.org.

Michelle Cohn, Chun-Yen Chen, and Zhou Yu. 2019 A large-scale user study of an Alexa Prize chatbot: Effect of TTS dynamism on perceived quality of social dialog. In Proceedings of the 20th Annual SIGdial Meeting on Discourse and Dialogue, pages 293 306, Stockholm, Sweden. Association for Computational Linguistics.

Yuning Du, Chenxia Li, Ruoyu Guo, Xiaoting Yin, Weiwei Liu, Jun Zhou, Yifan Bai, Zilin Yu, Yehua Yang, Qingqing Dang, et al. 2020. PP-OCR: A Practical Ultra lightweight OCR system. ArXiv preprint, abs/2009.09941.

Jason Eppink. 2014. A brief history of the gif (so far). Journal of visual culture, 13(3):298-306.
Wong Erinn. 2019. Digital blackface: How 21st century internet language reinforces racism.

Hao Fang, Hao Cheng, Maarten Sap, Elizabeth Clark, Ari Holtzman, Yejin Choi, Noah A. Smith, and Mari Ostendorf. 2018. Sounding board: A user-centric and content-driven social chatbot. In Proceedings of the 2018 Conference of the North American Chapter of the Association for Computational Linguistics: Demonstrations, pages 96-100, New Orleans, Louisiana. Association for Computational Linguistics.

Jianfeng Gao, Michel Galley, and Lihong Li. 2019 Neural Approaches to Conversational AI: Question Answering, Task-oriented Dialogues and Social Chatbots. Now Foundations and Trends.

Marjan Ghazvininejad, Chris Brockett, Ming-Wei Chang, Bill Dolan, Jianfeng Gao, Wen-tau Yih, and Michel Galley. 2018. A knowledge-grounded neural conversation model. In Proceedings of the ThirtySecond AAAI Conference on Artificial Intelligence, (AAAI-18), the 30th innovative Applications of Artificial Intelligence (IAAI-18), and the 8th AAAI Symposium on Educational Advances in Artificial Intelligence (EAAI-18), New Orleans, Louisiana, USA, February 2-7, 2018, pages 5110-5117. AAAI Press.

Carla F Griggio, Joanna Mcgrenere, and Wendy E Mackay. 2019. Customizations and expression breakdowns in ecosystems of communication apps. Proceedings of the ACM on Human-Computer Interaction, 3(CSCW):1-26.

Tim Highfield and Tama Leaver. 2016. Instagrammatics and digital methods: Studying visual social media, from selfies and gifs to memes and emoji. Communication research and practice, 2(1):47-62.

Chung Hoon Hong, Yuan Liang, Sagnik Sinha Roy, Arushi Jain, Vihang Agarwal, Ryan Draves, Zhizhuo Zhou, William Chen, Yujian Liu, Martha Miracky, et al. 2020. Audrey: A personalized open-domain conversational bot. In Alexa Prize Proceedings.

Pingping Huang, Jianhui Huang, Yuqing Guo, Min Qiao, and Yong Zhu. 2019. Multi-grained attention with object-level grounding for visual question answering. In Proceedings of the 57th Annual Meeting of the Association for Computational Linguistics, pages 3595-3600, Florence, Italy. Association for Computational Linguistics.

Michimasa Inaba and Kenichi Takahashi. 2016. Neural utterance ranking model for conversational dialogue systems. In Proceedings of the 17th Annual Meeting of the Special Interest Group on Discourse and Dialogue, pages 393-403, Los Angeles. Association for Computational Linguistics.

Jialun "Aaron" Jiang, Jed R Brubaker, and Casey Fiesler. 2017. Understanding diverse interpretations of animated GIFs. In Proceedings of the $2017 \mathrm{CHI}$ Conference Extended Abstracts on Human Factors in Computing Systems, pages 1726-1732. 
Jialun "Aaron" Jiang, Casey Fiesler, and Jed R Brubaker. 2018. "The Perfect One" Understanding Communication Practices and Challenges with Animated GIFs. Proceedings of the ACM on humancomputer interaction, 2(CSCW):1-20.

Daniel Jurafsky, Elizabeth Shriberg, Barbara Fox, and Traci Curl. 1998. Lexical, prosodic, and syntactic cues for dialog acts. In Discourse Relations and Discourse Markers.

Mahmoud Khademi. 2020. Multimodal neural graph memory networks for visual question answering. In Proceedings of the 58th Annual Meeting of the Association for Computational Linguistics, pages 71777188, Online. Association for Computational Linguistics.

Ryan Kiros, Ruslan Salakhutdinov, and Richard S Zemel. 2014. Unifying visual-semantic embeddings with multimodal neural language models. ArXiv preprint, abs/1411.2539.

Artie Konrad, Susan C Herring, and David Choi. 2020. Sticker and emoji use in facebook messenger: implications for graphicon change. Journal of ComputerMediated Communication, 25(3):217-235.

Vaibhav Kumar and Jamie Callan. 2020. Making information seeking easier: An improved pipeline for conversational search. In Findings of the Association for Computational Linguistics: EMNLP 2020, pages 3971-3980, Online. Association for Computational Linguistics.

Kuang-Huei Lee, X. Chen, G. Hua, H. Hu, and Xiaodong He. 2018. Stacked cross attention for imagetext matching. ArXiv preprint, abs/1803.08024.

Xiujun Li, Xi Yin, Chunyuan Li, Pengchuan Zhang, Xiaowei Hu, Lei Zhang, Lijuan Wang, Houdong Hu, Li Dong, Furu Wei, et al. 2020. Oscar: Objectsemantics aligned pre-training for vision-language tasks. In European Conference on Computer Vision, pages 121-137. Springer.

John Savery Madden. 2018. The Phenomenological Exploration of Animated GIF Use in ComputerMediated Communication. Ph.D. thesis, University of Oklahoma.

Luke Melas-Kyriazi, Alexander Rush, and George Han. 2018. Training for diversity in image paragraph captioning. In Proceedings of the 2018 Conference on Empirical Methods in Natural Language Processing, pages 757-761, Brussels, Belgium. Association for Computational Linguistics.

Kate M Miltner and Tim Highfield. 2017. Never gonna GIF you up: Analyzing the cultural significance of the animated GIF. Social Media+ Society, 3(3):2056305117725223.

Dat Quoc Nguyen, Thanh Vu, and Anh Tuan Nguyen. 2020. BERTweet: A pre-trained language model for English tweets. In Proceedings of the 2020
Conference on Empirical Methods in Natural Language Processing: System Demonstrations, pages 914, Online. Association for Computational Linguistics.

Tianrui Niu, Fangxiang Feng, Lingxuan Li, and Xiaojie Wang. 2020. Image synthesis from locally related texts. In Proceedings of the 2020 International Conference on Multimedia Retrieval, pages 145-153.

Silvia Pareti and Tatiana Lando. 2018. Dialog intent structure: A hierarchical schema of linked dialog acts. In Proceedings of the Eleventh International Conference on Language Resources and Evaluation (LREC 2018), Miyazaki, Japan. European Language Resources Association (ELRA).

Gustavo Penha and Claudia Hauff. 2021. On the calibration and uncertainty of neural learning to rank models for conversational search. In Proceedings of the 16th Conference of the European Chapter of the Association for Computational Linguistics: Main Volume, pages 160-170, Online. Association for Computational Linguistics.

Alec Radford, Jong Wook Kim, Chris Hallacy, Aditya Ramesh, Gabriel Goh, Sandhini Agarwal, Girish Sastry, Amanda Askell, Pamela Mishkin, Jack Clark, et al. 2021. Learning transferable visual models from natural language supervision. ArXiv preprint, $\mathrm{abs} / 2103.00020$.

Ashwin Ram, Rohit Prasad, Chandra Khatri, Anu Venkatesh, Raefer Gabriel, Qing Liu, Jeff Nunn, Behnam Hedayatnia, Ming Cheng, Ashish Nagar, et al. 2018. Conversational ai: The science behind the alexa prize. ArXiv preprint, abs/1801.03604.

Aditya Ramesh, Mikhail Pavlov, Gabriel Goh, Scott Gray, Mark Chen, Rewon Child, Vedant Misra, Pamela Mishkin, Gretchen Kruegerand Sandhini Agarwal, and Ilya Sutskever. 2021. DALL-E: Creating images from text. https://openai.com/ blog/dall-e/.

Daniel J Russo, Benjamin Van Roy, Abbas Kazerouni, Ian Osband, and Zheng Wen. 2018. A tutorial on thompson sampling. Foundations and Trends ${ }^{\circledR}$ in Machine Learning, 11(1):1-96.

Konstantinos Sechidis, Grigorios Tsoumakas, and Ioannis Vlahavas. 2011. On the stratification of multilabel data. Machine Learning and Knowledge Discovery in Databases, pages 145-158.

Piyush Sharma, Nan Ding, Sebastian Goodman, and Radu Soricut. 2018. Conceptual captions: A cleaned, hypernymed, image alt-text dataset for automatic image captioning. In Proceedings of the 56th Annual Meeting of the Association for Computational Linguistics (Volume 1: Long Papers), pages 2556-2565, Melbourne, Australia. Association for Computational Linguistics. 
Piotr Szymański and Tomasz Kajdanowicz. 2017. A network perspective on stratification of multi-label data. In First International Workshop on Learning with Imbalanced Domains: Theory and Applications, pages 22-35. PMLR.

Mingxing Tan and Quoc V. Le. 2019. Efficientnet: Rethinking model scaling for convolutional neural networks. In Proceedings of the 36th International Conference on Machine Learning, ICML 2019, 915 June 2019, Long Beach, California, USA, volume 97 of Proceedings of Machine Learning Research, pages 6105-6114. PMLR.

Ying Tang and Khe Foon Hew. 2019. Emoticon, emoji, and sticker use in computer-mediated communication: A review of theories and research findings. International Journal of Communication, 13:27.

Andree Thieltges, Florian Schmidt, and Simon Hegelich. 2016. The devil's triangle: Ethical considerations on developing bot detection methods. In 2016 AAAI Spring Symposium Series.

Jackson Tolins and Patrawat Samermit. 2016. Gifs as embodied enactments in text-mediated conversation. Research on Language and Social Interaction, 49(2):75-91.

Liwei Wang, Yin Li, and Svetlana Lazebnik. 2016. Learning deep structure-preserving image-text embeddings. In 2016 IEEE Conference on Computer Vision and Pattern Recognition, CVPR 2016, Las Vegas, NV, USA, June 27-30, 2016, pages 5005-5013. IEEE Computer Society.

Zihao Wang, Xihui Liu, Hongsheng Li, Lu Sheng, Junjie Yan, Xiaogang Wang, and Jing Shao. 2019. CAMP: cross-modal adaptive message passing for text-image retrieval. In 2019 IEEE/CVF International Conference on Computer Vision, ICCV 2019, Seoul, Korea (South), October 27 - November 2, 2019, pages 5763-5772. IEEE.

Miaomiao Wen, Nancy Baym, Omer Tamuz, Jaime Teevan, Susan T Dumais, and Adam Kalai. 2015. Omg ur funny! computer-aided humor with an application to chat. In ICCC, pages 86-93.

Jun Xu, Haifeng Wang, Zheng-Yu Niu, Hua Wu, Wanxiang Che, and Ting Liu. 2020. Conversational graph grounded policy learning for open-domain conversation generation. In Proceedings of the 58th Annual Meeting of the Association for Computational Linguistics, pages 1835-1845, Online. Association for Computational Linguistics.

Tao Xu, Pengchuan Zhang, Qiuyuan Huang, Han Zhang, Zhe Gan, Xiaolei Huang, and Xiaodong He. 2018. Attngan: Fine-grained text to image generation with attentional generative adversarial networks. In 2018 IEEE Conference on Computer Vision and Pattern Recognition, CVPR 2018, Salt Lake City, UT, USA, June 18-22, 2018, pages 1316-1324. IEEE Computer Society.
Rui Yan, Yiping Song, and Hua Wu. 2016. Learning to respond with deep neural networks for retrievalbased human-computer conversation system. In Proceedings of the 39th International ACM SIGIR conference on Research and Development in Information Retrieval, SIGIR 2016, Pisa, Italy, July 17-21, 2016, pages 55-64. ACM.

Shi Yu, Zhenghao Liu, Chenyan Xiong, Tao Feng, and Zhiyuan Liu. 2021. Few-shot conversational dense retrieval. ArXiv preprint, abs/2105.04166.

Li Zhou, Jianfeng Gao, Di Li, and Heung-Yeung Shum. 2020. The design and implementation of Xiaolce, an empathetic social chatbot. Computational Linguistics, 46(1):53-93. 


\begin{tabular}{ll}
\hline \hline Category & Subcategory \\
\hline \hline Cartoons \& Comics & aqua teen hunger force \\
Celebrities & richard pryor \\
Reactions & angry \\
Emotions & happy \\
Anime & bleach \\
Art \& Design & psychedelic \\
Nature & sunrise \\
Transportation & bicycle \\
\hline \hline
\end{tabular}

Table 5: Examples of GIF categories on GIPHY

\section{A Additional Details on Model Training}

Following, we provide additional details on how each of the three models was trained.

\section{A.1 Tag-based Model}

EfficientNet-based Tag Classifier Gifs are reshaped to 224 by 224 pixel while keeping the aspect ratio by padding and normalized to a mean of 0.5 and standard deviation of 0.5 for each channel before feeding into the EfficientNet-based model. We selected unique GIFs from the finalized dataset that has at least one associated tag and using the iterative train test split on k-hot tag representation to select $5 \%$ of those GIFs for validation. The EfficientNet tag classifier was trained for 100 epochs on a batch size of 32, using AdamW optimizer with learning rate $1 \mathrm{e}-5$ and weight decay $1 \mathrm{e}-3$. The best validation performance was achieved at the 40th epoch with macro-f1 of 0.30 in predicting 241 multi-label classes. Early experiment shows that transformer encoder layer (macro-f1 of 0.30 ) out performs linear layer (macro-f1 of 0.19) in fusing multi-frame gif features on the development set, therefore transformer encoder layer is used to fuse features of different frames in our implementation. Tweet-to-tag classifier Using the finalized dataset mentioned in $\S 3$, we use tweet as input, and the $\mathrm{k}$-hot tag representation of that tweet instance as ground truth label to train the multi-label classifier along with the tweet encoder for 241 classes. Additionally, we filter out tweets from the finalized dataset that do not have corresponding twitter tags before training. The model with the best validation performance is selected to perform subsequent evaluation and field experiments. The tweet encoder was trained for 100 epochs with a batch size of 32. The learning rate was set to 1e-5 with 1e-3 weight decay using AdamW optimizer. The best validation macro-f1 was 0.07 achieved at the 70th epoch.

\section{A.2 CLIP variant}

The evaluation performance for model selection is measured by nDCG. For every tweet-gif pair in the validation set, we measure the top 30 predicted GIFs from the model using the tweet as input. The relevance of an occurring ground truth gif in the top 30 predictions given a tweet is set 1 for the nDCG calculation.

CLIP variant is trained on the same finalized dataset using contrastive loss. It was trained for 16 epochs with a batch size of 16 using AdamW optimizer of learning rate 1e-5 and weight decay $1 \mathrm{e}-3$. Best validation performance is achieved at epoch 6 with an nDCG value of 0.015 .

We replace the Transformer encoder layer with a linear Layer on Efficient GIF Encoder from Figure 3, and use this as our GIF Encoder for the CLIP variant. Image inputs to the GIF encoder are normalized following the official CLIP implementation.

\section{A.3 Pepe}

The PEPE model follows most configurations from the CLIP variant model, but replace the EffcientNet GIF encoder with an Oscar GIF encoder based on Oscar pre-trained multi-modal transformer $(\mathrm{Li}$ et al., 2020).

Extra metadata are extracted from GIFs in the finalized dataset for further training. Captions within the GIF are extracted using PaddleOCR (Du et al., 2020), and only extracted text with probability greater than 0.9 are kept as caption metadata.

Object tags and their corresponding features are extracted with bottom-up attention (Anderson et al., 2018) using py-bottom-up-attention package. Object instances are filtered to only keep instances that have a score higher than 0.5 , then object tags and their corresponding features are extracted from these instances. Final object features of dimension 2054 are obtained by concatenating feature output with dimension 2048 from Faster-RCNN with scaled box position coordinates of the object following ( $\mathrm{Li}$ et al., 2020).

The PEPE model is trained on the finalized dataset with extracted caption and object metadata. It was trained for 16 epochs with a batch size of 8 using AdamW optimizer of learning rate 1e-6 and weight decay 1e-3. Preprocessing for GIFs is 
Tweet-GIF Pairs

42, $\underline{096}, \underline{566}$ pairs

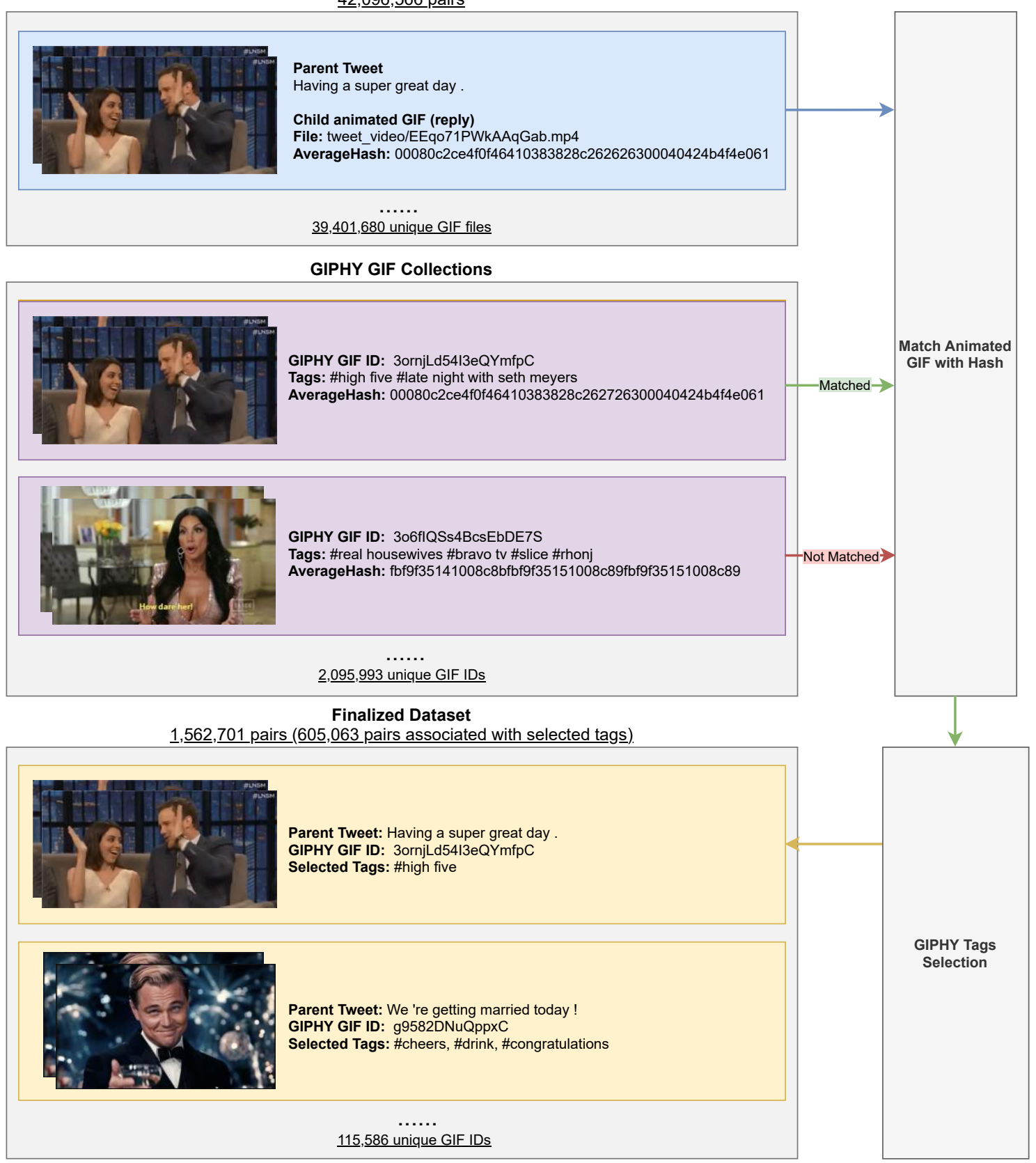

Figure 7: A diagram of the pipeline used to collect, canonicalize, and filter gif-reply data from Twitter. 


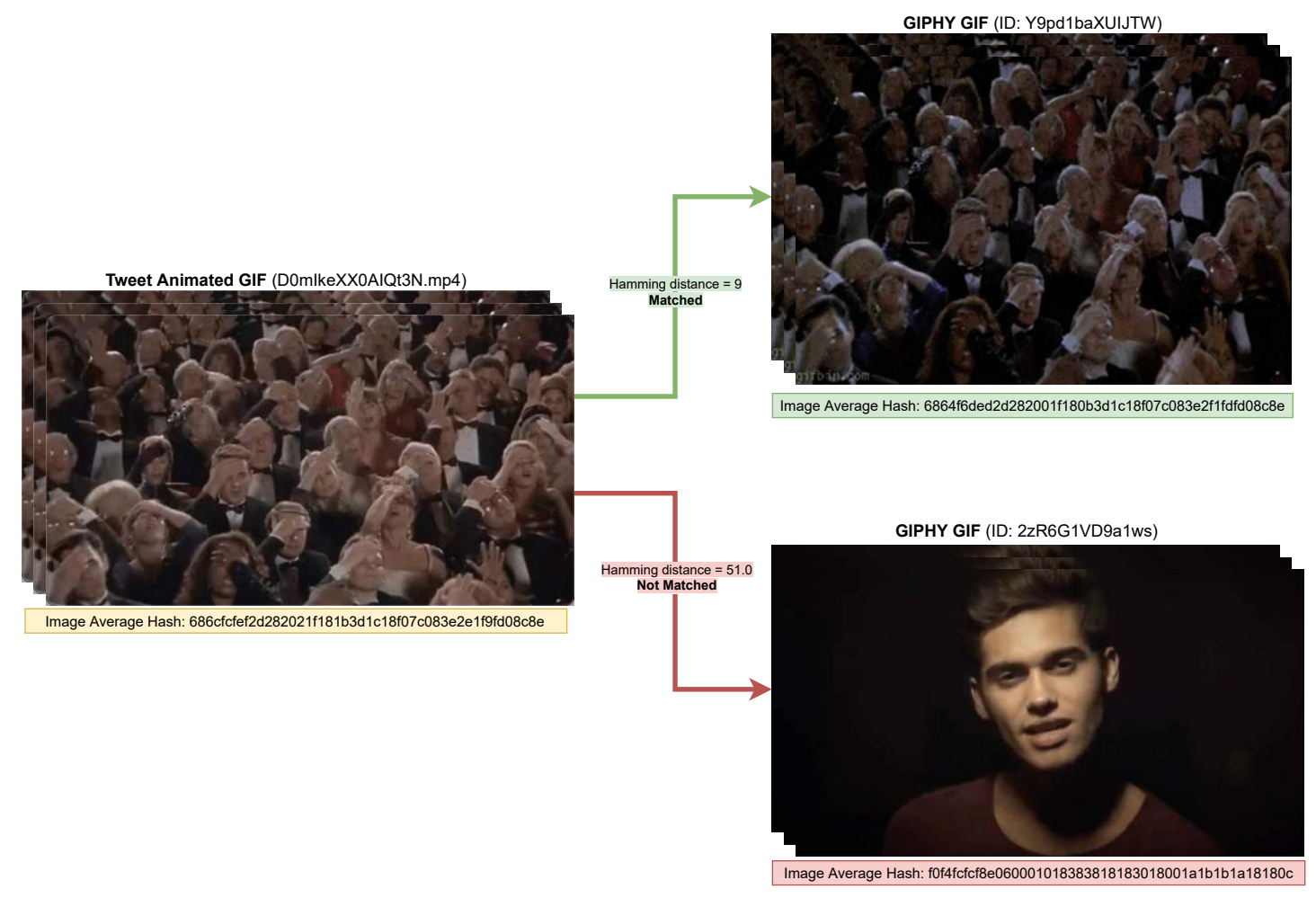

Figure 8: Matching Animated GIFs from Twitter with GIPHY gifs using Image Average Hash

the same as the Tag-based model. Max sequence length is set to 256 tokens for the Oscar transformer. Best evaluation performance is achieved at epoch 12 with an nDCG score of 0.007 .

\section{B GIF categories on GIPHY}

\begin{tabular}{ll}
\hline Category & Subcategory \\
\hline Reactions & what \\
Reactions & hair flip \\
Reactions & bored \\
Reactions & frown \\
Reactions & slow clap \\
Reactions & mic drop \\
Reactions & goodbye \\
Reactions & meh \\
Reactions & scared \\
Reactions & do not want \\
Reactions & confused \\
Reactions & drunk \\
Reactions & wow \\
Reactions & mad \\
Reactions & awesome \\
Reactions & please
\end{tabular}

Reactions thumbs down

Reactions frustrated

Reactions oh snap

Reactions disgusted

Reactions rejected

Reactions embarrassed

Reactions hug

Reactions yolo

Reactions interested

Reactions thank you

Reactions sarcastic

Reactions shocked

Reactions cool story bro

Reactions middle finger

Reactions you got this

Reactions whatever

Reactions omg

Reactions deal with it

Reactions sigh

Reactions oops

Reactions angry

Reactions finger guns

Reactions good luck 


\begin{tabular}{lc}
\hline \hline & Dependent variable: \\
\cline { 2 - 2 } & Gif reply score \\
\hline post score & $-0.0002^{* * *}(0.00003)$ \\
comment score & $0.001^{* * *}(0.0001)$ \\
CLIP variant model & $-0.161^{* * *}(0.058)$ \\
Distribution-sampling model & $0.057(0.056)$ \\
PEPE model & $0.223^{* * *}(0.051)$ \\
Tag-based model & $-0.017(0.055)$ \\
number of days after reply & $0.003^{* * *}(0.0005)$ \\
comment text polarity & $-0.039(0.058)$ \\
comment text subjectivity & $-0.033(0.052)$ \\
topic 0 (Politics related) & $0.078(0.155)$ \\
topic 1 (Family \& Pets related) & $0.300^{* *}(0.148)$ \\
topic 2 (Employment related) & $-0.119(0.184)$ \\
topic 3 (Social media related) & $0.140(0.165)$ \\
topic 4 (Transportation related) & $-0.172(0.188)$ \\
topic 5 (Food related) & $0.133(0.194)$ \\
topic 6 (COVID related) & $-0.082(0.200)$ \\
topic 7 (Entertainment related) & $-0.057(0.161)$ \\
topic 8 (People related) & $0.272(0.198)$ \\
comment is a question & $0.068(0.049)$ \\
length of parent comment & $-0.003(0.002)$ \\
intercept & $0.231^{* *}(0.115)$ \\
\hline Observations & 8,369 \\
Log Likelihood & $-14,899.820$ \\
$\theta$ & $0.548^{* * *}(0.013)$ \\
Akaike Inf. Crit. & $29,841.640$ \\
\hline \hline Note: & $* \mathrm{p}<0.1 ;{ }^{* *} \mathrm{p}<0.05 ;{ }^{* * *} \mathrm{p}<0.01$ \\
\end{tabular}

Table 6: Negative Binomial regression on score of the gif reply. The random-gif baseline is set as the reference category for model comparison. 


\begin{tabular}{|c|c|c|c|}
\hline Reactions & abandon thread & Reactions & judging you \\
\hline Reactions & excited & Transportation & truck \\
\hline Reactions & suspicious & Transportation & spaceship \\
\hline Reactions & win & Transportation & van \\
\hline Reactions & applause & Transportation & submarine \\
\hline Reactions & popcorn & Transportation & motorcycle \\
\hline Reactions & sleepy & Transportation & bmw \\
\hline Reactions & nod & Transportation & helicopter \\
\hline Reactions & awww & Transportation & chevrolet \\
\hline Reactions & disappointed & Transportation & volkswagen \\
\hline Reactions & ugh & Transportation & boat \\
\hline Reactions & laughing & Transportation & bus \\
\hline Reactions & oh no you didnt & Transportation & porsche \\
\hline Reactions & $\mathrm{smh}$ & Transportation & tank \\
\hline Reactions & agree & Transportation & audi \\
\hline Reactions & serious & Transportation & toyota \\
\hline Reactions & party hard & Transportation & airplane \\
\hline Reactions & shut up & Transportation & hovercraft \\
\hline Reactions & ok & Transportation & nissan \\
\hline Reactions & help & Transportation & bicycle \\
\hline Reactions & smile & Transportation & train \\
\hline Reactions & incredulous & Transportation & rocket \\
\hline Reactions & yawn & Transportation & yacht \\
\hline Reactions & idk & Transportation & ferrari \\
\hline Reactions & sexy & Transportation & honda \\
\hline Reactions & fist bump & Transportation & sailboat \\
\hline Reactions & dancing & Transportation & car \\
\hline Reactions & nom & Transportation & tesla \\
\hline Reactions & eww & Holidays & mardi gras \\
\hline Reactions & hello & Holidays & oktoberfest \\
\hline Reactions & not bad & Holidays & kwanzaa \\
\hline Reactions & success & Holidays & fathers day \\
\hline Reactions & burn & Holidays & fourth of july \\
\hline Reactions & proud & Holidays & mothers day \\
\hline Reactions & i give up & Holidays & yom kippur \\
\hline Reactions & hearts & Holidays & st patricks day \\
\hline Reactions & pleased & Holidays & memorial day \\
\hline Reactions & fml & Holidays & cinco de mayo \\
\hline Reactions & sorry & Holidays & labor day \\
\hline Reactions & aroused & Holidays & rosh hashanah \\
\hline Reactions & happy dance & Holidays & new years \\
\hline Reactions & good job & Holidays & passover \\
\hline Reactions & wtf & Science & global warming \\
\hline Reactions & seriously & Science & astronomy \\
\hline Reactions & want & Science & physics \\
\hline Reactions & rage & Science & laser \\
\hline Reactions & table flip & Science & stars \\
\hline Reactions & love & Science & robot \\
\hline Reactions & amused & Science & atoms \\
\hline Reactions & flirt & Science & meteor \\
\hline
\end{tabular}




\begin{tabular}{|c|c|c|c|}
\hline Science & bubbles & Greetings & happy anniversary \\
\hline Science & medicine & Greetings & hey \\
\hline Science & nebula & Greetings & welcome \\
\hline Science & technology & Greetings & cheers \\
\hline Science & mathematics & Greetings & best friends \\
\hline Science & chemistry & $\mathrm{TV}$ & workaholics \\
\hline Science & biology & TV & succession \\
\hline Science & planets & $\mathrm{TV}$ & blackish \\
\hline Science & magnets & TV & shark tank \\
\hline Science & molecules & TV & big brother \\
\hline Science & asteroids & TV & vanderpump rules \\
\hline Science & space & TV & afv \\
\hline Science & bill nye & TV & twin peaks \\
\hline Science & engineering & TV & its always sunny in \\
\hline Science & diy & & philadelphia \\
\hline Science & nuclear & $\mathrm{TV}$ & real housewives of new \\
\hline Science & computers & & york city \\
\hline Fashion \& Beauty & chanel & TV & seinfeld \\
\hline Fashion \& Beauty & alexander mcqueen & TV & american horror story \\
\hline Fashion \& Beauty & model & TV & modern family \\
\hline Fashion \& Beauty & victorias secret & TV & poldark \\
\hline Fashion \& Beauty & prada & $\mathrm{TV}$ & stranger things \\
\hline Fashion \& Beauty & karlie kloss & TV & law and order svu \\
\hline Fashion \& Beauty & jessica stam & TV & big mouth \\
\hline Fashion \& Beauty & emily ratajkowski & TV & greys anatomy \\
\hline Fashion \& Beauty & miranda kerr & TV & bachelor in paradise \\
\hline Fashion \& Beauty & kate upton & $\mathrm{TV}$ & i love lucy \\
\hline Fashion \& Beauty & louis vuitton & TV & the voice \\
\hline Fashion \& Beauty & makeup & TV & boy meets world \\
\hline Fashion \& Beauty & kate moss & TV & the bachelorette \\
\hline Fashion \& Beauty & cara delevingne & TV & new girl \\
\hline Fashion \& Beauty & runway & $\mathrm{TV}$ & south park \\
\hline Fashion \& Beauty & jourdan dunn & TV & saturday night live \\
\hline Fashion \& Beauty & julia nobis & TV & saved by the bell \\
\hline Fashion \& Beauty & jewelry & TV & real housewives of new jer- \\
\hline Fashion \& Beauty & beauty & & sey \\
\hline Fashion \& Beauty & chanel iman & Food \& Drink & pancakes \\
\hline Fashion \& Beauty & christian dior & Food \& Drink & sandwich \\
\hline Fashion \& Beauty & marc jacobs & Food \& Drink & happy hour \\
\hline Fashion \& Beauty & shoes & Food \& Drink & sushi \\
\hline Fashion \& Beauty & dress & Food \& Drink & steak \\
\hline Fashion \& Beauty & gucci & Food \& Drink & pasta \\
\hline Greetings & get well & Food \& Drink & french toast \\
\hline Greetings & bye & Food \& Drink & mimosa \\
\hline Greetings & im out & Food \& Drink & tea \\
\hline Greetings & sympathy & Food \& Drink & whiskey \\
\hline Greetings & thank you & Food \& Drink & pickle \\
\hline Greetings & new baby & Food \& Drink & cake \\
\hline Greetings & im sorry & Food \& Drink & egg roll \\
\hline Greetings & congratulations & Food \& Drink & broccoli \\
\hline
\end{tabular}


Food \& Drink

Food \& Drink

Food \& Drink

Food \& Drink

Food \& Drink

Food \& Drink

Food \& Drink

Food \& Drink

Food \& Drink

Food \& Drink

Food \& Drink

Food \& Drink

Food \& Drink

Food \& Drink

Food \& Drink

Food \& Drink

Food \& Drink

Food \& Drink

Gaming

Gaming

Gaming

Gaming

Gaming

Gaming

Gaming

Gaming

Gaming

Gaming

Gaming

Gaming

Gaming

Gaming

Gaming

Gaming

Gaming

Gaming

Gaming

Gaming

Gaming

Gaming

Gaming

Gaming

Gaming

Gaming

Gaming

Gaming

Gaming

Gaming

Gaming

Gaming vodka

bread

cookie

taco

cheese

brunch

french fries

apple

orange fruit

brownies

wine

ham

salad

pie

soda

beer

burrito

banana

donkey kong

max payne

gears of war

streets of rage

starfox

metroid

sega

prince of persia

sprites

final fantasy

wolfenstein $3 d$

call of duty

earthbound

tetris

video game physics

nintendo

pacman

game boy

tomb raider

super mario

sonic the hedgehog

the last of us

half life

dead space

nes

super nintendo

animal crossing

n64

atari

the sims

bioshock

portal
Gaming destiny the game

Gaming 8bit

Gaming galaga

Gaming kirby

Gaming mortal kombat

Gaming starcraft

Gaming duck hunt

Gaming skyrim

Gaming grand theft auto

Gaming mods

Gaming metal gear solid

Gaming world of warcraft

Gaming super smash bros

Interests new york city

Interests vampire

Interests ballet

Interests summer

Interests butt

Interests winter

Interests tumblr

Interests roller coaster

Interests robot

Interests iphone

Interests work

Interests theme park

Interests zombie

Interests party

Interests baby

Interests lgbt

Interests internet

Interests boy

Interests alien

Interests girl

Interests vacation

Interests boobs

Interests ghost

Interests autumn

Interests spring

Interests clown

Celebrities jean claude van damme

Celebrities paul scheer

Celebrities denzel washington

Celebrities bryan cranston

Celebrities chris pratt

Celebrities johnny depp

Celebrities stephen colbert

Celebrities emma watson

Celebrities macaulay culkin

Celebrities heath ledger

Celebrities jim gaffigan 
Celebrities

Celebrities

Celebrities

Celebrities

Celebrities

Celebrities

Celebrities

Celebrities

Celebrities

Celebrities

Celebrities

Celebrities

Celebrities

Celebrities

Celebrities

Celebrities

Celebrities

Celebrities

Celebrities

Celebrities

Celebrities

Celebrities

Celebrities

Celebrities

Celebrities

Celebrities

Celebrities

Celebrities

Celebrities

Celebrities

Celebrities

Celebrities

Celebrities

Celebrities

Celebrities

Celebrities

Celebrities

Celebrities

Celebrities

Celebrities

Celebrities

Celebrities

Celebrities

Celebrities

Celebrities

Celebrities

Celebrities

Celebrities

Celebrities

Celebrities mr. $\mathrm{t}$

danny mcbride

michael fassbender

seth rogen

elijah wood

jon hamm

tom hanks

kate upton

arnold schwarzenegger

tom hiddleston

al pacino

sean connery

javier bardem

ken jeong

will smith

maya rudolph

jack mcbrayer

leonardo dicaprio

clint eastwood

robert downey jr

michael ian black

adrien brody

tom hardy

joseph gordon levitt

mark ruffalo

adam baldwin

rebel wilson

jim carrey

melissa mccarthy

ashley benson

rob huebel

julianne moore

hayden panettiere

anna kendrick

will forte

ryan gosling

andrew garfield

nick offerman

weird al yankovic

will arnett

bruce lee

christian bale

paul dano

eddie murphy

sam rockwell

mike tyson

jude law

rooney mara

adam sandler

chris hemsworth
Celebrities kristen wiig

Celebrities james franco

Celebrities adam scott

Celebrities seth green

Celebrities jeremy renner

Celebrities morgan freeman

Celebrities bradley cooper

Celebrities dave chappelle

Celebrities rachel mccadams

Celebrities nicolas cage

Celebrities megan fox

Celebrities robert redford

Celebrities elizabeth banks

Celebrities liam neeson

Celebrities willem dafoe

Celebrities jonah hill

Celebrities michael cera

Celebrities charlie sheen

Celebrities emma roberts

Celebrities jon stewart

Celebrities patton oswalt

Celebrities samuel 1 jackson

Celebrities alison brie

Celebrities matt lucas

Celebrities ellen page

Celebrities amanda bynes

Celebrities jake gyllenhaal

Celebrities rob lowe

Celebrities steve carell

Celebrities conan obrien

Celebrities cillian murphy

Celebrities mindy kaling

Celebrities ben stiller

Celebrities john travolta

Celebrities gary oldman

Celebrities amy poehler

Celebrities ian somerhalder

Celebrities richard pryor

Celebrities bruce willis

Celebrities daniel day lewis

Celebrities chuck norris

Celebrities ed helms

Celebrities don cheadle

Celebrities michael caine

Celebrities george carlin

Celebrities alia shawkat

Celebrities emma stone

Celebrities adam devine

Celebrities larry david

Celebrities taylor kitsch 


\begin{tabular}{|c|c|c|c|}
\hline Celebrities & matthew perry & Actions & falling \\
\hline Celebrities & dave franco & Actions & smoking \\
\hline Celebrities & harrison ford & Actions & flirting \\
\hline Celebrities & olivia munn & Actions & dancing \\
\hline Celebrities & emily blunt & Actions & breaking up \\
\hline Celebrities & mila kunis & Actions & drinking \\
\hline Celebrities & ru paul & Actions & fainting \\
\hline Celebrities & jason bateman & Emotions & shocked \\
\hline Celebrities & anne hathaway & Emotions & bored \\
\hline Celebrities & tracy morgan & Emotions & unimpressed \\
\hline Celebrities & natalie portman & Emotions & sick \\
\hline Celebrities & brad pitt & Emotions & stressed \\
\hline Celebrities & tom cruise & Emotions & nervous \\
\hline Celebrities & sylvester stallone & Emotions & sad \\
\hline Celebrities & tina fey & Emotions & relaxed \\
\hline Celebrities & dolph lundgren & Emotions & sassy \\
\hline Celebrities & tony hale & Emotions & tired \\
\hline Celebrities & donald glover & Emotions & reaction \\
\hline Celebrities & paul rudd & Emotions & hungry \\
\hline Celebrities & angelina jolie & Emotions & scared \\
\hline Celebrities & scarlett johansson & Emotions & angry \\
\hline Celebrities & david cross & Emotions & drunk \\
\hline Celebrities & alec baldwin & Emotions & lonely \\
\hline Celebrities & david duchovny & Emotions & pain \\
\hline Celebrities & will ferrell & Emotions & excited \\
\hline Celebrities & chris rock & Emotions & happy \\
\hline Celebrities & adam brody & Emotions & surprised \\
\hline Celebrities & jennifer lawrence & Emotions & inspired \\
\hline Celebrities & aubrey plaza & Emotions & suspicious \\
\hline Celebrities & jackie chan & Emotions & frustrated \\
\hline Celebrities & alexa chung & Emotions & love \\
\hline Celebrities & ricky gervais & Emotions & embarrassed \\
\hline Celebrities & jessica walter & Emotions & disappointed \\
\hline Actions & cooking & Sports & hockey \\
\hline Actions & fighting & Sports & rugby \\
\hline Actions & smiling & Sports & nhl \\
\hline Actions & laughing & Sports & rock climbing \\
\hline Actions & dreaming & Sports & diving \\
\hline Actions & crying & Sports & formula one \\
\hline Actions & spinning & Sports & rowing \\
\hline Actions & tossing drink & Sports & skydiving \\
\hline Actions & sleeping & Sports & $\mathrm{mma}$ \\
\hline Actions & eating & Sports & lacrosse \\
\hline Actions & sneezing & Sports & ufc \\
\hline Actions & singing & Sports & volleyball \\
\hline Actions & pout & Sports & softball \\
\hline Actions & slapping & Sports & $\mathrm{mlb}$ \\
\hline Actions & finger guns & Sports & martial arts \\
\hline Actions & running & Sports & horse racing \\
\hline Actions & swimming & Sports & skiing \\
\hline
\end{tabular}




\begin{tabular}{|c|c|c|c|}
\hline Sports & swimming & Adjectives & slow motion \\
\hline Sports & roller skating & Adjectives & cute \\
\hline Sports & football & Adjectives & cold \\
\hline Sports & tennis & Adjectives & funny \\
\hline Sports & nba & Adjectives & weird \\
\hline Sports & boxing & Adjectives & trippy \\
\hline Sports & parkour & Adjectives & black and white \\
\hline Sports & nascar & Adjectives & pretty \\
\hline Sports & golf & Adjectives & scary \\
\hline Art \& Design & art & Adjectives & creepy \\
\hline Art \& Design & typography & Adjectives & hd \\
\hline Art \& Design & illustration & Animals & lizard \\
\hline Art \& Design & transparent & Animals & meerkat \\
\hline Art \& Design & glitch & Animals & otter \\
\hline Art \& Design & pixel & Animals & cow \\
\hline Art \& Design & morph & Animals & caterpillar \\
\hline Art \& Design & black and white & Animals & koala \\
\hline Art \& Design & geometry & Animals & corgi \\
\hline Art \& Design & collage & Animals & penguin \\
\hline Art \& Design & architecture & Animals & duck \\
\hline Art \& Design & psychedelic & Animals & elephant \\
\hline Art \& Design & $3 \mathrm{~d}$ & Animals & raccoon \\
\hline Art \& Design & mash up & Animals & hippo \\
\hline Art \& Design & photography & Animals & kangaroo \\
\hline Art \& Design & loop & Animals & chicken \\
\hline Art \& Design & cinemagraph & Animals & monkey \\
\hline Art \& Design & sculpture & Animals & ferret \\
\hline Art \& Design & timelapse & Animals & seal \\
\hline Art \& Design & design & Animals & owl \\
\hline Art \& Design & animation & Animals & jellyfish \\
\hline Memes & sips tea & Animals & bulldog \\
\hline Memes & steal yo girl & Animals & crab \\
\hline Memes & arthur & Animals & butterfly \\
\hline Memes & crying dawson & Animals & giraffe \\
\hline Memes & confused & Animals & panda \\
\hline Memes & deal with it & Animals & pig \\
\hline Memes & like a boss & Animals & red panda \\
\hline Memes & hair flip & Animals & grumpy cat \\
\hline Memes & forever alone & Animals & sheep \\
\hline Memes & look at all the fucks i give & Animals & turtle \\
\hline Memes & cuca & Animals & wolf \\
\hline Memes & judge judy & Animals & lion \\
\hline Memes & feels & Animals & bird \\
\hline Memes & fail & Animals & hamster \\
\hline Memes & dank memes & Animals & polar bear \\
\hline Adjectives & vintage & Animals & goat \\
\hline Adjectives & sexy & Animals & whale \\
\hline Adjectives & bright & Animals & mouse \\
\hline Adjectives & dark & Animals & camel \\
\hline Adjectives & hot & Animals & chihuahua \\
\hline
\end{tabular}




\begin{tabular}{|c|c|}
\hline Animals & skunk \\
\hline Animals & squirrel \\
\hline Animals & frog \\
\hline Animals & horse \\
\hline Animals & pug \\
\hline Animals & tiger \\
\hline Animals & unicorn \\
\hline Animals & bear \\
\hline Animals & poodle \\
\hline Movies & the fifth element \\
\hline Movies & the breakfast club \\
\hline Movies & addams family \\
\hline Movies & breakfast at tiffanys \\
\hline Movies & cry baby \\
\hline Movies & donnie darko \\
\hline Movies & waynes world \\
\hline Movies & say anything \\
\hline Movies & the godfather \\
\hline Movies & blue velvet \\
\hline Movies & the princess bride \\
\hline Movies & clueless \\
\hline Movies & ghostbusters \\
\hline Movies & spiderman \\
\hline Movies & sixteen candles \\
\hline Movies & ace ventura \\
\hline Movies & the blues brothers \\
\hline Movies & fight club \\
\hline Movies & indiana jones \\
\hline Movies & the notebook \\
\hline Movies & get out \\
\hline Movies & the matrix \\
\hline Movies & star wars \\
\hline Movies & night of the living dead \\
\hline Movies & the shining \\
\hline Movies & 500 days of summer \\
\hline Movies & bladerunner \\
\hline Movies & elf \\
\hline Movies & the big lebowski \\
\hline Movies & some like it hot \\
\hline Movies & american psycho \\
\hline Movies & easy rider \\
\hline Movies & reservoir dogs \\
\hline Movies & texas chainsaw massacre \\
\hline Movies & the avengers \\
\hline Movies & beetlejuice \\
\hline Movies & labyrinth \\
\hline Movies & scarface \\
\hline Movies & spring breakers \\
\hline Movies & rocky \\
\hline Movies & pretty in pink \\
\hline
\end{tabular}

\begin{tabular}{|c|c|}
\hline Movies & the dark knight \\
\hline Movies & citizen kane \\
\hline Movies & edward scissorhands \\
\hline Movies & kill bill \\
\hline Movies & casablanca \\
\hline Movies & pulp fiction \\
\hline Movies & terminator \\
\hline Movies & zoolander \\
\hline Movies & bridesmaids \\
\hline Movies & dodgeball \\
\hline Movies & heathers \\
\hline Movies & lost boys \\
\hline Movies & the goonies \\
\hline Movies & hocus pocus \\
\hline Movies & the hangover \\
\hline Identity & native american \\
\hline Identity & muslim \\
\hline Identity & love is love \\
\hline Identity & bisexual \\
\hline Identity & asian \\
\hline Identity & times up \\
\hline Identity & queer \\
\hline Identity & non binary \\
\hline Identity & gay \\
\hline Identity & lesbian \\
\hline News \& Politics & republican \\
\hline News \& Politics & cory booker \\
\hline News \& Politics & economy \\
\hline News \& Politics & irs \\
\hline News \& Politics & democrat \\
\hline News \& Politics & supreme court \\
\hline News \& Politics & bernie sanders \\
\hline News \& Politics & bill clinton \\
\hline News \& Politics & kamala harris \\
\hline News \& Politics & julian castro \\
\hline News \& Politics & white house \\
\hline News \& Politics & senate \\
\hline News \& Politics & joe biden \\
\hline News \& Politics & president \\
\hline News \& Politics & tax day \\
\hline News \& Politics & elizabeth warren \\
\hline News \& Politics & pete buttigieg \\
\hline News \& Politics & protest \\
\hline News \& Politics & climate change \\
\hline News \& Politics & nancy pelosi \\
\hline News \& Politics & congress \\
\hline News \& Politics & rbg \\
\hline News \& Politics & taxes \\
\hline Cartoons \& Comics & snow white \\
\hline Cartoons \& Comics & peter pan \\
\hline
\end{tabular}


Cartoons \& Comics

Cartoons \& Comics

Cartoons \& Comics

Cartoons \& Comics

Cartoons \& Comics

Cartoons \& Comics

Cartoons \& Comics

Cartoons \& Comics

Cartoons \& Comics

Cartoons \& Comics

Cartoons \& Comics

Cartoons \& Comics

Cartoons \& Comics

Cartoons \& Comics

Cartoons \& Comics

Cartoons \& Comics

Cartoons \& Comics

Cartoons \& Comics

Cartoons \& Comics

Cartoons \& Comics

Cartoons \& Comics

Cartoons \& Comics

Cartoons \& Comics

Cartoons \& Comics

Cartoons \& Comics

Cartoons \& Comics

Cartoons \& Comics

Cartoons \& Comics

Cartoons \& Comics

Cartoons \& Comics

Cartoons \& Comics

Cartoons \& Comics

Cartoons \& Comics

Cartoons \& Comics

Cartoons \& Comics

Cartoons \& Comics

Cartoons \& Comics

Cartoons \& Comics

Cartoons \& Comics

Cartoons \& Comics

Cartoons \& Comics

Cartoons \& Comics

Cartoons \& Comics

Cartoons \& Comics

Cartoons \& Comics

Cartoons \& Comics

Cartoons \& Comics

Cartoons \& Comics

Cartoons \& Comics doug

mulan

harvey birdman

the critic

hotel transylvania

gi joe

wile e coyote

popeye

regular show

aeon flux

the little mermaid

fosters home for imagi-

nary friends

animaniacs

gumby

adult swim

the jetsons

muppet babies

beavis and butthead

archie comics

mickey mouse

captain planet

peanuts

ren and stimpy

underdog

george of the jungle

gravity falls

grinch who stole christmas

mr magoo

top cat

dexters laboratory

tangled

betty boop

king of the hill

pink panther

tailspin

tweety bird

disney

sleeping beauty

aladdin

toy story

alvin and the chipmunks

teen titans

tom and jerry

minnie mouse

my little pony

the incredibles

pinocchio

rockos modern life

jem and the holograms
Cartoons \& Comics

Cartoons \& Comics

Cartoons \& Comics

Cartoons \& Comics

Cartoons \& Comics

Cartoons \& Comics

Cartoons \& Comics

Cartoons \& Comics

Cartoons \& Comics

Cartoons \& Comics

Cartoons \& Comics

Cartoons \& Comics

Cartoons \& Comics

Cartoons \& Comics

Cartoons \& Comics

Cartoons \& Comics

Cartoons \& Comics

Cartoons \& Comics

Cartoons \& Comics

Cartoons \& Comics

Cartoons \& Comics

Cartoons \& Comics

Cartoons \& Comics

Cartoons \& Comics

Cartoons \& Comics

Cartoons \& Comics

Cartoons \& Comics

Cartoons \& Comics

Cartoons \& Comics

Cartoons \& Comics

Cartoons \& Comics

Cartoons \& Comics

Cartoons \& Comics

Cartoons \& Comics

Cartoons \& Comics

Cartoons \& Comics

Cartoons \& Comics

Cartoons \& Comics

Cartoons \& Comics

Cartoons \& Comics

Cartoons \& Comics

Cartoons \& Comics

Cartoons \& Comics

Cartoons \& Comics

Cartoons \& Comics

Cartoons \& Comics

Cartoons \& Comics

Cartoons \& Comics

Cartoons \& Comics the flintstones

garfield

looney tunes

calvin and hobbes

batman

rugrats

home movies

scooby doo

speed racer

the venture bros

daffy duck

wall e

cars

101 dalmatians

beauty and the beast

porky pig

schoolhouse rock

rocky and bullwinkle

sealab 2021

hey arnold

josie and the pussycats

arthur

aqua teen hunger force

magical game time

space ghost

cartoon network

family guy

the lion king

winnie the pooh

phinas and ferb

homestuck

daria

fat albert

the oatmeal

yogi bear

fantasia

bambi

samurai jack

the powerpuff girls

cyanide and happiness

teenage mutant ninja tur-

tles

pocahontas

voltron

south park

finding nemo

metalocaypse

dreamworks

alice in wonderland

johnny bravo 


\begin{tabular}{|c|c|}
\hline Decades & $80 s$ \\
\hline Decades & vintage \\
\hline Decades & $30 \mathrm{~s}$ \\
\hline Decades & $60 s$ \\
\hline Decades & $50 \mathrm{~s}$ \\
\hline Decades & $70 \mathrm{~s}$ \\
\hline Decades & $40 \mathrm{~s}$ \\
\hline Decades & $90 \mathrm{~s}$ \\
\hline Decades & $20 \mathrm{~s}$ \\
\hline Weird & $80 \mathrm{~s}$ \\
\hline Weird & vintage \\
\hline Weird & ghost \\
\hline Weird & zombie \\
\hline Weird & morph \\
\hline Weird & psychedelic \\
\hline Weird & vampire \\
\hline Weird & alien \\
\hline Weird & $90 \mathrm{~s}$ \\
\hline Weird & robot \\
\hline Weird & clown \\
\hline Stickers & cat stickers \\
\hline Stickers & excited stickers \\
\hline Stickers & love stickers \\
\hline Stickers & animatedtext stickers \\
\hline Stickers & emoji stickers \\
\hline Stickers & weird stickers \\
\hline Stickers & high five stickers \\
\hline Stickers & birthday stickers \\
\hline Stickers & party stickers \\
\hline Stickers & cheeseburger stickers \\
\hline Stickers & happy stickers \\
\hline Stickers & dinosaur stickers \\
\hline Nature & sun \\
\hline Nature & waves \\
\hline Nature & wind \\
\hline Nature & river \\
\hline Nature & mist \\
\hline Nature & desert \\
\hline Nature & moon \\
\hline Nature & waterfall \\
\hline Nature & stars \\
\hline Nature & tsunami \\
\hline Nature & coral \\
\hline Nature & glacier \\
\hline Nature & weather \\
\hline Nature & beach \\
\hline Nature & sunrise \\
\hline Nature & comet \\
\hline Nature & ocean \\
\hline Nature & ice \\
\hline
\end{tabular}

\begin{tabular}{|c|c|}
\hline Nature & crystals \\
\hline Nature & forest \\
\hline Nature & sunset \\
\hline Nature & fire \\
\hline Nature & lava \\
\hline Nature & reef \\
\hline Nature & tornado \\
\hline Nature & northern lights \\
\hline Nature & landscape \\
\hline Nature & prairie \\
\hline Nature & night \\
\hline Nature & plants \\
\hline Nature & cave \\
\hline Nature & trees \\
\hline Nature & constellations \\
\hline Nature & clouds \\
\hline Nature & hurricane \\
\hline Nature & sand \\
\hline Nature & mushrooms \\
\hline Nature & snow \\
\hline Nature & geyser \\
\hline Nature & lake \\
\hline Nature & mountains \\
\hline Nature & smoke \\
\hline Nature & rainbow \\
\hline Music & action bronson \\
\hline Music & adele \\
\hline Music & frank ocean \\
\hline Music & kendrick lamar \\
\hline Music & the beatles \\
\hline Music & mc hammer \\
\hline Music & zayn malik \\
\hline Music & nicki minaj \\
\hline Music & backstreet boys \\
\hline Music & lizzo \\
\hline Music & $\mathrm{cl}$ \\
\hline Music & snoop dogg \\
\hline Music & madonna \\
\hline Music & usher \\
\hline Music & vampire weekend \\
\hline Music & the rolling stones \\
\hline Music & g dragon \\
\hline Music & jennifer lopez \\
\hline Music & janet jackson \\
\hline Music & destinys child \\
\hline Music & lady gaga \\
\hline Music & jay z \\
\hline Music & elvis presley \\
\hline Music & bruno mars \\
\hline Music & cardi b \\
\hline
\end{tabular}




\begin{tabular}{ll} 
Music & tlc \\
Music & david bowie \\
Music & coldplay \\
Music & kpop \\
Music & missy elliott \\
Music & solange \\
Music & whitney houston \\
Music & carrie underwood \\
Music & shakira \\
Music & britney spears \\
Music & lil nas x \\
Music & mariah carey \\
Music & selena gomez \\
Anime & samurai champloo \\
Anime & fullmetal alchemist \\
Anime & bleach \\
Anime & spaceship battleship yam- \\
& ato \\
Anime & manga \\
Anime & hetalia \\
Anime & princess mononoke \\
Anime & my neighbor totoro \\
Anime & cowboy bebop \\
Anime & kawaii \\
Anime & kiba \\
Anime & berserk \\
Anime & evangelion \\
Anime & black lagoon \\
Anime & inuyasha \\
Anime & ninja scroll \\
Anime & sakura \\
Anime & hayao miyazaki \\
Anime & cardcaptor sakura \\
Anime & rock lee \\
Anime & code geass \\
Anime & kakashi hatake \\
Anime & hinata hyuga \\
Anime & death note \\
Anime & gundam \\
\hline &
\end{tabular}

\section{List of selected tags from GIPHY}

adorable, agreed, amazing, amused, angry, annoyed, anxiety, anxious, applause, approval, approve, aw, awesome, awkward, bad, beautiful, best wishes, blank stare, blink, blush, bored, bow, bravo, but why, buy, bye, captivated, celebrate, cheeky, cheering, cheers, clap, come on, comic, compliment, compliments, concerned, confused, congratulations, cool, crazy, creeping, cringe, crushing, cry, curtsy, cute, damn, dance, dancing, deadpan stare, debate, depressed, dickhead, disagree, dis- appointed, disapprove, disbelief, disgust, dislike, diss, divertente, dont care, doubt, doubtful, drink, drinking, drunk, dubious, dying, eating, eating popcorn, embarassed, engrossed, ennui, excited, face palm, faint, fingers crossed, flirt, flushed, freaking out, frustrated, fuck, fun, funny, gagging, get well, glare, good luck, gossip, grateful, gratitude, great, great job, grin, hahahah, happy, happy dance, head shake, hide, high five, hilarious, honestly, hope, horror, hugs, hugs love, hysterical, ill, impressed, incredulous, insult, interested, interesting, judge, judging you, just, keep going, kiss, laugh, leaving, lets go, lies, like, looking, looking around, love you, lovely, luv u, luv you, mad, mind blown, mock, motivational, moved, muah, much appreciated, nah, nasty, need, nervous, nice one, no, nod, not amused, not funny, not interested, oh shit, overwhelmed, panic, partying, perfect, pissed, please, pleased, pointing, praise, pray, pregnant, proud, pumped, questioning, raises hand, realization, relief, respect, reunited, roast, roll eyes, sad, sadness, salute, sarcastic, savage, scared, scary, screaming, secret, seriously, sexy, shame, shock, shook, shrug, shut up, shy, sigh, sips tea, sitting, sleepy, sloth, smart, smile, smug, sobbing, sorpren, sorry, spit, stoked, stressed, stunned, success, sudden realization, surprise, suspicious, sweating, swoon, swooning, take notes, tantrum, tears, thank, think, thirsty, thumbs down, thumbs up, tired, too funny, touched, unamused, unbelievable, uncomfortable, unhappy, unimpressed, unsure, upset, vomit, waiting, wave, weary, weird, whatever, will, wince, wink, wrestling, yawn, yell, yes, yum

\section{List of filtering keywords on Imgur experiment}

depression, depressing, mental, health, death, dead, alcohol, alcoholism, weed, drugs, addiction, covid, beer, stoned, black, white, arabic, hispanic, latino, latina, latinx, police, cop, racism, racists, race, sexism, sexist, sexy, armed, overthrow, government, republican, democrats, maga, liberal, liberals, conservative, conservatives, offender, victim, disability, disabled, jerking, PD, gun, shots, fired, cops, officer, officers, killing, murder, murdered, kill, kills, killed, murders, shoot, taser, bystander, trigger, handgun, pansexual, sexuality, homosexual, gay, lesbian, corona, virus, coronavirus, vaccine, vaccinated, viruses, vaccination, die, fascist, fascists, antifa, sharia, islam, islamic, christian, jewish, muslim, blasphemy, blasphemic, death, conviction, 
church, priest, pastor, religious, religion, sharia, shia, sunni, judge, bible, qaran, torah, hindu, hindus, christians, jew, jews, muslims, islamist, execute, murder, captive, captives, malpractice, insurance, insured, threat, threatening, war, troops, violence, fighting, conflict, medicine, prescription, drug, dying, hospice, life, doctor, hospital, nurse, pedophiles, pedophile, bitch, republicans, democrat, coup, tax, recession, pedo, criminal, criminals, politician, politicians, health, healthcare, america, american, voter, voting, votes, vote, voters, citizen, immigrants, immigrant, citizens, candian, canada, eu, european, trump, red, blue, cancer, slavery, slaves, slave, disease, sickness, sorry, nazi, nazis, death, pro-death, pro-life, profile, abortion, aborted, aborting, victims, jail, whore, slut, rape, raped, raping, behead, beheadings, beheaded, torture, tortured, torturing, taliban, afghanistan, soldier, soldiers, kabul 


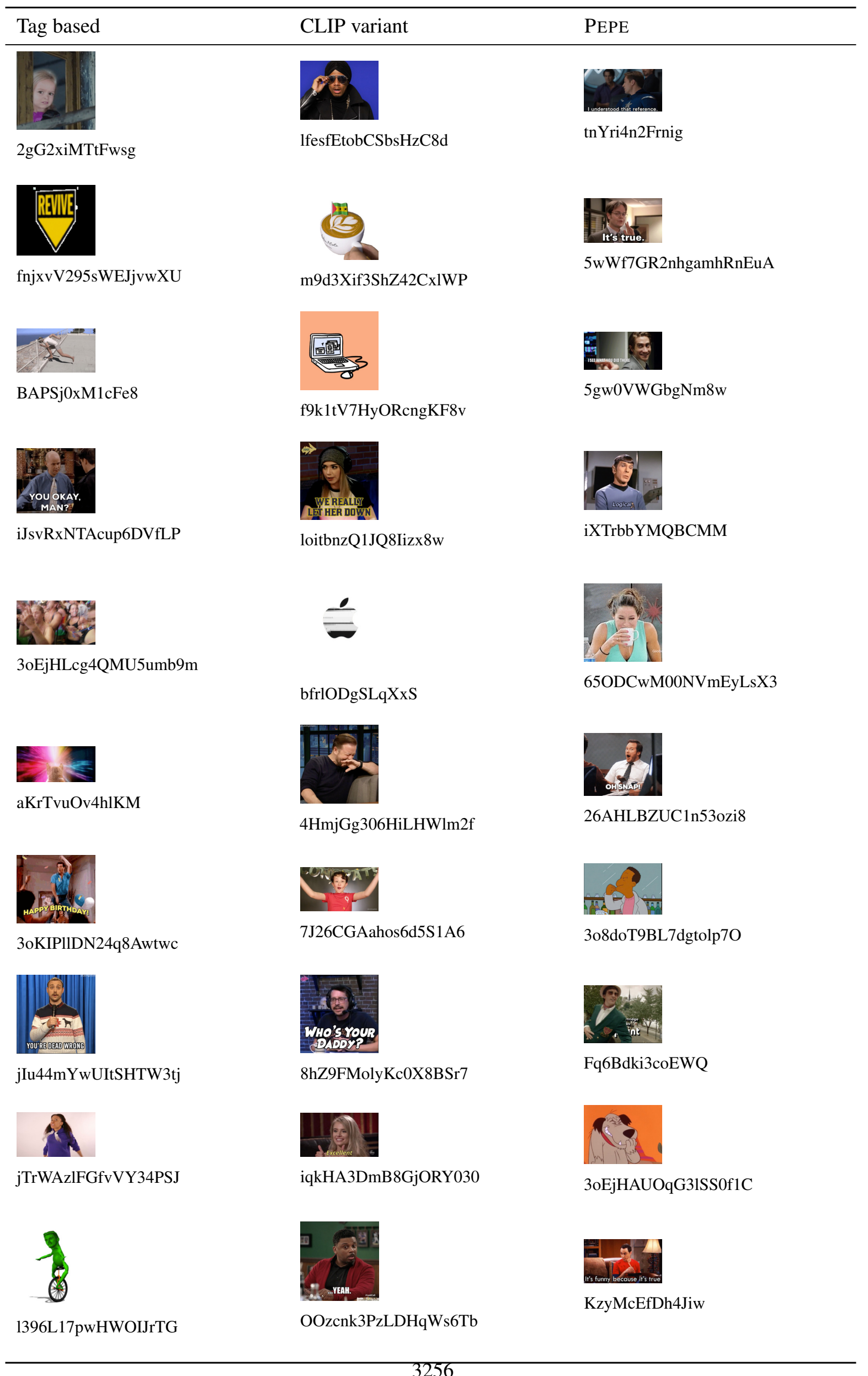

Table 7: Examples of top 10 most frequently used gifs across all models in the RCT. Click an image to view the gif on Giphy. Images are ordered from most-used (top) to tenth-most (bottom). 


\begin{tabular}{lc}
\hline \hline & Dependent variable: \\
\cline { 2 - 2 } & Cumulative number of replies received \\
\hline gif reply score & $0.096^{* * *}(0.010)$ \\
post score & $-0.0004^{* * *}(0.0001)$ \\
comment score & $0.0002(0.0002)$ \\
CLIP variant model & $-0.196(0.152)$ \\
distribution-sampling model & $-0.664^{* * *}(0.160)$ \\
PEPE model & $-0.450^{* * *}(0.138)$ \\
Tag-based model & $-0.195(0.146)$ \\
number of days after reply & $-0.001(0.001)$ \\
comment text polarity & $0.048(0.164)$ \\
comment text subjectivity & $-0.055(0.147)$ \\
topic 0 (Politics related) & $-0.275(0.430)$ \\
topic (Family \& Pets related) & $-0.264(0.412)$ \\
topic 2 (Employment related) & $-1.182^{* *}(0.549)$ \\
topic 3 (Social media related) & $1.381^{* * *}(0.421)$ \\
topic 4 (Transportation related) & $-0.021(0.514)$ \\
topic 5 (Food related) & $-0.896(0.567)$ \\
topic 6 (COVID related) & $-0.459(0.564)$ \\
topic 7 (Entertainment related) & $-0.529(0.452)$ \\
topic 8 (People related) & $-1.776^{* * *}(0.647)$ \\
comment is a question & $0.114(0.133)$ \\
length of parent comment & $0.0003(0.007)$ \\
intercept & $-1.877^{* * *}(0.313)$ \\
\hline Observations & 8,369 \\
Log Likelihood & $-2,466.965$ \\
$\theta$ & $0.143^{* * *}(0.013)$ \\
Akaike Inf. Crit. & $4,977.930$ \\
\hline \hline Note: & p $<0.1 ; * * \mathrm{p}<0.05 ; * * * \mathrm{p}<0.01$ \\
&
\end{tabular}

Table 8: Negative Binomial regression on cumulative number of replies received. The random-gif baseline is set as the reference category for model comparison.

\begin{tabular}{ccl}
\hline \hline Topic & Dirichlet parameter & Keywords \\
\hline 0 & 0.1172 & people fuck trump shit make thing country n't vote fucking \\
1 & 0.20164 & good time love kid make cat dog day year guy \\
2 & 0.09554 & pay work money people make job year buy time company \\
3 & 0.11245 & post make read people good time thing imgur video work \\
4 & 0.06541 & car live year drive day place time road city back \\
5 & 0.05672 & eat make food good water drink taste cheese pizza coffee \\
6 & 0.06662 & people covid die vaccine life make work problem mask n't \\
7 & 0.0888 & movie play game good watch show love great time song \\
8 & 0.02752 & wear mask red shirt woman hair white man hat black \\
9 & 0.14292 & back make put hand time guy car head thing big \\
\hline \hline
\end{tabular}

Table 9: Topic modeling keywords for Imgur Comments 